\title{
TRAVELING WAVE SOLUTIONS FOR SYSTEMS OF ODEs ON A TWO-DIMENSIONAL SPATIAL LATTICE*
}

\author{
JOHN W. CAHN ${ }^{\dagger}$, JOHN MALLET-PARET ${ }^{\ddagger}$, AND ERIK S. VAN VLECK ${ }^{\S}$
}

\begin{abstract}
We consider infinite systems of ODEs on the two-dimensional integer lattice, given by a bistable scalar ODE at each point, with a nearest neighbor coupling between lattice points. For a class of ideal nonlinearities, we obtain traveling wave solutions in each direction $e^{i \theta}$, and we explore the relation between the wave speed $c$, the angle $\theta$, and the detuning parameter $a$ of the nonlinearity. Of particular interest is the phenomenon of "propagation failure," and we study how the critical value $a=a^{*}(\theta)$ depends on $\theta$, where $a^{*}(\theta)$ is defined as the value of the parameter $a$ at which propagation failure (that is, wave speed $c=0$ ) occurs. We show that $a^{*}: \mathbb{R} \rightarrow \mathbb{R}$ is continuous at each point $\theta$ where $\tan \theta$ is irrational, and is discontinuous where $\tan \theta$ is rational or infinite.
\end{abstract}

Key words. traveling waves, propagation failure, anisotropy, Allen-Cahn equation

AMS subject classifications. 35K57, 73D99

PII. S0036139996312703

1. Introduction. In the study of crystal growth in material science, there is often an underlying spatial lattice which plays an important role in the evolution and dynamics of the system. Anisotropy is a central feature in such problems [5], [37], and a microscopic lattice structure can be a source of anisotropy which is observable on a macroscopic scale. For example, wave motion in a lattice may exhibit a velocity which is direction dependent; in addition, the lattice may cause a freezing or "pinning" of the wave motion, which does not occur for such motion in a continuum. These phenomena were described in the context of crystal growth by Gibbs [16, p. 324] almost 120 years ago and more recently examined by Cahn [3] for diffuse interfaces.

Systems of differential equations with an underlying lattice structure occur in mathematical models in many different scientific disciplines. Besides material science (see, for example, [4]), we mention biology [20], [39], and pattern recognition [12], [13]. See also the survey papers [8], [9], [21], and [24], for numerous additional references.

In this paper we study an idealized system of lattice differential equations, which exhibits all of the above phenomena, and which lends itself to rigorous mathematical analysis of these phenomena. In particular, we consider the infinite system of ODEs

$$
\dot{u}_{i, j}=\alpha(\Delta u)_{i, j}-f\left(u_{i, j}\right), \quad(i, j) \in \mathbb{Z}^{2},
$$

on the two-dimensional integer lattice $\mathbb{Z}^{2}$. Here each variable $u_{i, j}=u_{i, j}(t)$ is real valued, $\alpha>0$ is a parameter, $f: \mathbb{R} \rightarrow \mathbb{R}$ is a given function, and $\Delta$ denotes the

* Received by the editors December 2, 1996; accepted for publication (in revised form) November 19, 1997; published electronically November 18, 1998.

http://www.siam.org/journals/siap/59-2/31270.html

$\dagger$ Material Science and Engineering Laboratory, National Institute of Standards and Technology, Gaithersburg, MD 20760 (cahn@enh.nist.gov). The research of this author was partially supported by ARPA contract 70NANB8H0860.

${ }^{\ddagger}$ Division of Applied Mathematics, Brown University, Providence, RI 02912-9000 (jmp@cfm. brown.edu). The research of this author was partially supported by NSF grant DMS-93-10328, by ARO contract DAAH04-93-G-0198, and by ONR contract N00014-92-J-1481.

$\S$ Department of Mathematical and Computer Sciences, Colorado School of Mines, Golden, CO 80401 (erikvv@poincare.mines.edu). The research of this author was partially supported by NSF grant DMS-95-05049 and by NIST contract 43NANB714674. 
discrete Laplacian, defined as

$$
(\Delta u)_{i, j}=u_{i+1, j}+u_{i-1, j}+u_{i, j+1}+u_{i, j-1}-4 u_{i, j} .
$$

Typically one takes a function $f$ with a cubic-like shape (a double well potential)-for example,

$$
f(u)=u(u-a)(u-1),
$$

where $a \in(0,1)$ is a so-called "detuning" parameter. If $f$ is locally Lipschitz, then the initial value problem for (1.1) is well posed in $l^{\infty}\left(\mathbb{Z}^{2}\right)$, and we obtain local existence and uniqueness of solutions in that space in both forward and backward time. (Quite generally, if $D$ is any countable set, we let $l^{\infty}(D)$ denote the Banach space of all bounded functions $\varphi: D \rightarrow \mathbb{R}$ or $\varphi: D \rightarrow \mathbb{C}$, with the supremum norm.)

Let us briefly examine the (linearized) stability of certain equilibrium solutions of (1.1). If $f(b)=0$ for some $b \in \mathbb{R}$, then the spatially homogeneous element $u^{0} \in l^{\infty}\left(\mathbb{Z}^{2}\right)$ defined by $u_{i, j}^{0}=b$ for all $(i, j) \in \mathbb{Z}^{2}$ is an equilibrium of (1.1). The linearized stability of (1.1) at this equilibrium is determined from the spectrum of $L=\alpha \Delta-f^{\prime}(b)$ considered as an operator in $l^{\infty}\left(\mathbb{Z}^{2}\right)$. Let us write the discrete Laplacian $\Delta$ in (1.2) as $\Delta=S_{H}+S_{H}^{-1}+S_{V}+S_{V}^{-1}-4 I$, where $S_{H}$ and $S_{V}$ denote the horizontal and vertical shift operators on $l^{\infty}\left(\mathbb{Z}^{2}\right)$, namely $\left(S_{H} u\right)_{i, j}=u_{i+1, j}$ and $\left(S_{V} u\right)_{i, j}=u_{i, j+1}$. We have that $\operatorname{spec}\left(S_{H}\right)=\operatorname{spec}\left(S_{V}\right)=S^{1}$, where $S^{1}=\{\lambda \in \mathbb{C}|| \lambda \mid=1\}$ is the unit circle in the complex plane, and where $\operatorname{spec}(T)$ denotes the spectrum of an operator $T$. Therefore,

$$
\operatorname{spec}\left(S_{H}+S_{H}^{-1}\right)=\operatorname{spec}\left(S_{V}+S_{V}^{-1}\right)=[-2,2]
$$

by the spectral mapping theorem. We also note that if $X$ is any Banach space, and $T_{1}$ and $T_{2}$ are two bounded linear operators on $X$ which commute, $T_{1} T_{2}=T_{2} T_{1}$, then

$$
\operatorname{spec}\left(T_{1}+T_{2}\right) \subseteq \operatorname{spec}\left(T_{1}\right)+\operatorname{spec}\left(T_{2}\right),
$$

where here $A_{1}+A_{2}$ denotes the set of all sums $a_{1}+a_{2}$, with $a_{1} \in A_{1}$ and $a_{2} \in A_{2}$, for subsets $A_{1}, A_{2} \subseteq \mathbb{C}$. This result follows from the general theory of commutative Banach algebras; see, in particular, Theorem 11.23 in [33]. As $S_{H}+S_{H}^{-1}$ and $S_{V}+S_{V}^{-1}$ commute, we therefore have from (1.4) that

$$
\operatorname{spec}(\Delta) \subseteq[-8,0] .
$$

In fact, one sees that equality holds in (1.5). Given any $\lambda \in[-8,0]$, there exist $\zeta_{1}, \zeta_{2} \in S^{1}$ such that $\lambda=\zeta_{1}+\zeta_{1}^{-1}+\zeta_{2}+\zeta_{2}^{-1}-4$. Thus the element $u \in l^{\infty}\left(\mathbb{Z}^{2}\right)$ given by $u_{i, j}=\zeta_{1}^{i} \zeta_{2}^{j}$ satisfies $\Delta u=\lambda u$, and $\operatorname{so} \lambda \in \operatorname{spec}(\Delta)$.

It follows now that $\operatorname{spec}(L)$ is the interval

$$
\operatorname{spec}(L)=\left\{\begin{array}{l}
{\left[-8 \alpha-f^{\prime}(b),-f^{\prime}(b)\right] \text { for } \alpha \geq 0,} \\
{\left[-f^{\prime}(b),-8 \alpha-f^{\prime}(b)\right] \text { for } \alpha \leq 0 .}
\end{array}\right.
$$

Thus we have linearized stability if $f^{\prime}(b)>\max \{0,-8 \alpha\}$ holds, and linearized instability if on the other hand $f^{\prime}(b)<\max \{0,-8 \alpha\}$. In particular, if $f^{\prime}(b)>0$ (for example with $f$ as in (1.3), with $a \in(0,1)$, and $b=0$ or 1$)$, the equilibrium $b$ is stable for any $\alpha \geq 0$. In fact, such an equilibrium is stable for $\alpha<0$ sufficiently near zero, namely for $\alpha>-f^{\prime}(b) / 8$. 
By analogy with the related PDE

$$
u_{t}=\alpha \Delta u-f(u), \quad x \in \mathbb{R}^{2},
$$

for $u=u(t, x)$, where here $\Delta$ denotes the usual Laplacian $\Delta=D_{x_{1}}^{2}+D_{x_{2}}^{2}$, one expects that traveling wave solutions will play an important role in understanding the dynamics of (1.1). However, one soon sees that the structure of such solutions for the lattice system (1.1) is richer and much more complex than for the PDE (1.6). The study of traveling wave solutions of (1.6) leads to the second order ODE

$$
-c \varphi^{\prime}=\alpha \varphi^{\prime \prime}-f(\varphi)
$$

for $\varphi: \mathbb{R} \rightarrow \mathbb{R}$, with appropriate boundary conditions for $\varphi( \pm \infty)$. (See in particular the pioneering work in [15] on this problem.) On the other hand, for lattice equations such as (1.1), one typically instead obtains a differential-difference equation.

More specifically, consider the analogue of (1.1)

$$
\dot{u}_{i}=\alpha\left(u_{i+1}-2 u_{i}+u_{i-1}\right)-f\left(u_{i}\right), \quad i \in \mathbb{Z},
$$

in one space dimension. Following [44], consider solutions $u_{i}(t)$ of the form

$$
u_{i}(t)=\varphi(i-c t),
$$

for some unknown function $\varphi: \mathbb{R} \rightarrow \mathbb{R}$ and unknown wave speed $c \in \mathbb{R}$. For $c \neq 0$, we easily see that (1.9) is equivalent to the requirement that $u_{i}(t+1 / c)=u_{i-1}(t)$ for all $i$ and $t$. Quite generally, any solution (1.8) of the form (1.9) may be considered a traveling wave solution. Substitution of (1.9) into (1.8) yields the differential-difference equation

$$
-c \varphi^{\prime}(\xi)=\alpha(\varphi(\xi+1)-2 \varphi(\xi)+\varphi(\xi-1))-f(\varphi(\xi))
$$

for $\varphi(\xi)$. If we assume that $f$ has a cubic-like shape, as for example (1.3), with $f(0)=f(1)=0$, then it is natural to impose the boundary conditions

$$
\varphi(-\infty)=0, \quad \varphi(\infty)=1,
$$

indicating that $u_{i} \rightarrow 0$ or 1 as $i \rightarrow-\infty$ or $\infty$, respectively. As noted above, if $\alpha \geq 0$ then the two spatially homogeneous equilibria $b=0,1$ are stable.

In contrast to the second order equation (1.7), the differential-difference equation (1.10) is a genuinely infinite-dimensional problem. As described in the work of Rustichini [34], [35], the natural setting for an equation such as (1.10) is the Banach space $C[-1,1]$ of continuous functions on $[-1,1]$. This is in the spirit of the general treatment [18] of Hale and Verduyn Lunel, in which differential equations with retarded arguments (such as involving $\varphi(\xi-1)$ but not $\varphi(\xi+1)$ ) are placed in a function space setting. Moreover, while a great deal is known about retarded differential equations, with the exception of Rustichini's work very little of any general theory addresses the so-called "mixed"-type equations (1.10) in which both forward $\xi+1$ and backward $\xi-1$ shifts of the argument $\xi$ appear. Nevertheless, existence of solutions for a class of problems exemplified by (1.10), (1.11) is proved in [44] and [19] (see also [43]). More recently, a systematic study of the general theory of such mixed equations, and of the global structure of the solutions, was initiated in [22], [23], and this should provide 
additional tools for the analysis of the present problems. Results on problems with Fisher-type (monostable) nonlinearities are given in [39] and more recently in [42] and [45]. Finally, for results on traveling waves in a circular ring see [1], [40], and [41], and for traveling waves for discrete-time problems see [11].

An important qualitative difference between traveling wave solutions of the two systems (1.6) and (1.8) is the occurrence of "propagation failure" in the discrete system (1.8). To describe this phenomenon, which was studied in [20] (see also [32]), first consider the PDE (1.6) and the related ODE (1.7). Let $f$ be the cubic nonlinearity (1.3), and impose the boundary conditions (1.11). Associated with the second order system (1.7), which is a damped Hamiltonian system, is the energy function $E: \mathbb{R}^{2} \rightarrow$ $\mathbb{R}$ given by

$$
E\left(\varphi, \varphi^{\prime}\right)=\frac{\alpha}{2}\left(\varphi^{\prime}\right)^{2}-F(\varphi), \quad \frac{d F}{d \varphi}=f .
$$

If $\varphi(\xi)$ satisfies (1.7), then

$$
\frac{d E}{d \xi}=-c\left(\varphi^{\prime}(\xi)\right)^{2}
$$

holds, for $E=E\left(\varphi(\xi), \varphi^{\prime}(\xi)\right)$ evaluated along the solution. (We caution the reader that the function $E$ is related to, but different from, the energy functional

$$
V=\int_{\Omega} \frac{\alpha}{2}|\nabla u|^{2}-F(u) d x
$$

associated with the original PDE (1.6) on a bounded domain $\Omega \subseteq \mathbb{R}^{2}$. If $u(t, x)$ satisfies (1.6) on $\Omega$, with appropriate boundary conditions, then

$$
\frac{d V}{d t}=-\int_{\Omega} u_{t}^{2} d x \leq 0
$$

holds, for $V$ evaluated along this solution.) Observe that the quantity (1.13) is everywhere nonpositive if $c \geq 0$, and is everywhere nonnegative if $c \leq 0$. Also note that for a solution $\varphi$ of (1.7) satisfying the boundary conditions (1.11), the function $E$ in (1.12) has the limits $-F(0)$ and $-F(1)$ respectively, as $\xi \rightarrow-\infty$ and $\xi \rightarrow \infty$. By examining the values of $F(0)$ and $F(1)$ and by considering the sign of $c$, one easily sees from $(1.12),(1.13)$ that for any such solution, the quantities $a-1 / 2$ (where $a$ is the parameter in the function $f$ ) and $c$ have the same sign. In particular, when $a=1 / 2$ one obtains a standing wave, namely, a solution with $c=0$; but for any $a \neq 1 / 2$, we necessarily have a nonzero wave speed $c \neq 0$.

In contrast to the above scenario, the situation for the lattice system (1.8), with the differential-difference equation (1.10), with (1.11), is very different. Here there is no discrete analogue of the energy function $E$ for (1.10) (despite the fact that there is an analogue of the functional $V$ for (1.1)). We will show that typically there exists a nontrivial interval $|a-1 / 2| \leq \gamma$, with $\gamma>0$, in which $c=0$ must hold for any traveling wave solution of $(1.10),(1.11)$. Thus, in order to obtain a wave traveling with nonzero speed $c \neq 0$ (propagation), it is necessary to choose the parameter $a$ at least a distance $\gamma$ away from the center value $1 / 2$, that is, one requires that $|a-1 / 2|>\gamma$. Throughout the interval $|a-1 / 2| \leq \gamma$ the standing wave fails to propagate along the lattice.

An elementary argument based on the implicit function theorem partially confirms this phenomenon. Define a map

$$
G: l^{\infty}(\mathbb{Z}) \times \mathbb{R} \times \mathbb{R} \rightarrow l^{\infty}(\mathbb{Z})
$$


given in coordinate form by

$$
G(u, \alpha, a)_{i}=\alpha\left(u_{i+1}-2 u_{i}+u_{i-1}\right)-f\left(u_{i}, a\right),
$$

where we denote the dependence of the nonlinearity $f$ in (1.3) on $a$. Certainly $G\left(u^{0}, 0, a\right)=0$ for any $a$, where $u^{0} \in l^{\infty}(\mathbb{Z})$ denotes the element

$$
u_{i}^{0}=\left\{\begin{array}{cc}
0, & i<0, \\
1, & i \geq 0 .
\end{array}\right.
$$

Moreover, $G$ is Fréchet differentiable, and if $a \in(0,1)$, then its Fréchet derivative $D_{u} G\left(u^{0}, 0, a\right)$ with respect to its first argument is an isomorphism in $l^{\infty}(\mathbb{Z})$, being given by a diagonal operator with entries $-D_{u} f\left(u_{i}^{0}, a\right) \neq 0$. The implicit function theorem ensures that given any $a \in(0,1)$, then for $\alpha$ sufficiently near 0 there exists a solution $u^{*}=u^{*}(\alpha, a)$ near $u^{0}$ to the equation $G(u, \alpha, a)=0$. The solution $u^{*}$ thus corresponds to a standing wave, namely a solution of (1.10) with $c=0$. With additional arguments, one might expect to conclude monotonicity, $u_{i}^{*}<u_{i+1}^{*}$, of this solution in $i$ and also conclude that the boundary conditions (1.11) hold for this solution (which we do later, but for a different nonlinearity $f$ ). Thus, for any $a \in(0,1)$, one would have that $|a-1 / 2| \leq \gamma=\gamma(\alpha)$ for all sufficiently small $\alpha>0$. Note in particular this implies that $\gamma(\alpha) \rightarrow 1 / 2$ as $\alpha \rightarrow 0$. See [27] for further results in this spirit, where in particular uniform and explicit estimates on the range of $\alpha$ for which the implicit function theorem is valid are given.

We must add a cautionary note. For (1.10), (1.11), it is not obvious that a solution (even if monotone in $\xi$ ) is unique for a given $c$. In fact, it is not obvious for a given $\alpha$ and $a$ that the wave speed $c$ for which a solution exists is unique. One might expect that for a particular $\alpha$ and $a$, there may be several solutions of (1.10), (1.11) with either equal or different wave speeds $c$ and with either $c=0$ or $c \neq 0$ or both. And even if, for each $\alpha$ and $a$, there is a unique wave speed $c=c(\alpha, a)$, it is not obvious that this $c$ depends monotonically on $a$. In this paper we shall consider a particular $f$, related to but different from (1.3), and prove such uniqueness and monotonicity claims for traveling waves, which we construct in a fairly explicit fashion. In [23] such results are proved for a general class of $f$ including (1.3).

There is a further richness of structure that arises in problems on a two-dimensional lattice, stemming from the fact that one may consider different directions of motion of a traveling wave. As a result of the symmetry imposed by the lattice $\mathbb{Z}^{2}$, the existence and speed of a wave generally will depend on the direction $e^{i \theta}$ of motion, with a special role accorded those directions for which the slope $\tan \theta$ is rational. Let $\theta \in \mathbb{R}$ be given, denote

$$
\sigma=\sin \theta, \quad \kappa=\cos \theta
$$

and consider solutions of (1.1) of the form

$$
u_{i, j}(t)=\varphi(i \kappa+j \sigma-c t)
$$

for some $c \in \mathbb{R}$, where again $\varphi: \mathbb{R} \rightarrow \mathbb{R}$. We may consider solutions of the form (1.15) to be traveling waves on the lattice $\mathbb{Z}^{2}$, in the direction $e^{i \theta}$. Substitution of (1.15) into (1.1) leads to the equation

$$
(1.16)-c \varphi^{\prime}(\xi)=\alpha(\varphi(\xi+\kappa)+\varphi(\xi-\kappa)+\varphi(\xi+\sigma)+\varphi(\xi-\sigma)-4 \varphi(\xi))-f(\varphi(\xi)),
$$


and again, it is natural to impose the boundary conditions (1.11). Note that if $\theta$ is an integer multiple of $\pi / 2$, then (1.16) reduces to (1.10) considered above. On the other hand, if $\theta-\pi / 4$ is an integer multiple of $\pi / 2$, then under the change of variables $\psi(\xi)=\varphi\left(2^{-1 / 2} \xi\right),(1.16)$ is equivalent to the equation

$$
-2^{1 / 2} c \psi^{\prime}(\xi)=2 \alpha(\psi(\xi+1)-2 \psi(\xi)+\psi(\xi-1))-f(\psi(\xi)),
$$

which is the same as (1.10), but with rescaled parameters $c$ and $\alpha$. For other values of $\theta$, one does not expect any reduction of (1.16) to a simpler equation such as (1.10). Unfortunately, the results in [19] do not cover this extension of the equation (1.10) to (1.16), although one strongly expects analogues of their results to hold for a broad class of nonlinear $f$.

In section 6 the relation between the wave speed $c$ and detuning parameter $a$ is plotted graphically in Figure 1, for several choices of $\theta$, for the idealized piecewise linear nonlinearity (3.1), (3.2). Propagation failure is clearly visible on this graph as a jump discontinuity in $a$, at $c=0$. Also see Table 1 in section 4 , where $\gamma(\theta)$ is tabulated for various values of both $\theta$ and $\alpha$. In particular, the limits $\gamma \rightarrow 1 / 2$ as $\alpha \rightarrow 0$, and also $\gamma \rightarrow 0$ as $\alpha \rightarrow \infty$, are suggested. In fact, these limits are rigorously established in section 4. Extensive calculations of the wave speed $c$ as a function of $a, \alpha$, and $\theta$, as well as calculations of the wave profile $\varphi(\xi)$ itself for various $a, \alpha$, and $\theta$, are to be found in [14].

Let us summarize the contents of this paper. In section 2, we prove a general result about solutions of (1.1) which are monotone in a given direction $e^{i \theta}$, but which are not necessarily traveling wave solutions (1.15). In particular, we prove that if an initial condition is monotone in some direction, then so is the solution for all future time. This general result holds for any Lipschitz nonlinearity $f$.

Section 3 is devoted to a brief discussion of the technical aspects of discontinuous functions $f$, and the meaning of solutions of (1.1) for such $f$.

In section 4 , we obtain traveling wave solutions (1.15) with speed $c \neq 0$ for a particular piecewise linear $f$ with a discontinuity, in every direction $e^{i \theta}$. Although the mechanics of this involves straightforward applications of the Fourier transform, considerable care is required in order to justify our calculations. We prove various monotonicity properties of the solution here. In all of this analysis, it is necessary to employ some of the general machinery of dynamical systems, such as the Mel'nikov method. We also examine the range of the detuning parameter $a$ for which these solutions exist, as a function of $\theta$, and we observe the phenomenon of propagation failure. In particular, we note that the critical values $a^{*}=1 / 2 \pm \gamma(\theta)$ of the parameter $a$ at which propagation failure first occurs, are functions which are continuous in $\theta$ only at points such that the slope $\tan \theta$ is irrational, and are discontinuous at $\theta$ for which $\tan \theta$ is rational or infinite.

In section 5 , we construct traveling wave solutions with speed $c=0$, that is, equilibrium solutions, by taking limits $c \rightarrow 0$ of the solutions constructed in section 4 . We prove that these solutions are unique within a given class of monotone solutions, and also that they exhibit some rather subtle continuity properties (such as possessing a dense set of discontinuities whenever $\tan \theta$ is irrational). When $\tan \theta$ is rational or infinite, we obtain a finite number (two or three) of distinct equilibrium solutions, modulo translation on the lattice, while for $\tan \theta$ irrational, we obtain uncountably many such solutions.

Finally, in section 6 , we summarize our results, and broadly discuss some related topics. 
2. Monotonicity of solutions. In this section we consider the role of monotonicity of solutions. In particular, all the traveling wave solutions we construct will turn out to be increasing functions $\varphi(\xi)$ of the wave variable $\xi$. In order to underscore the importance of solutions with such monotonicity properties, we note here that if $\varphi$ is a solution of equation (1.16) for some $c \neq 0$, and if in addition $\varphi(\xi)$ is strictly increasing in $\xi$, both for $\xi \leq-T$, and for $\xi \geq T$, for some $T>0$, then in fact $\varphi(\xi)$ is strictly increasing in $\xi$ for all $\xi \in \mathbb{R}$. We do not present a formal proof of this fact, although we do prove a closely related result in Theorem 4.6, and we observe that a simple modification of this proof yields the claimed result.

Here we consider more general solutions of the lattice system (1.1), which may or may not be traveling waves (1.9), but which are monotone in the wave variable $\xi$. More precisely, let $\theta \in \mathbb{R}$ be fixed, and let $\sigma, \kappa$, be as in (1.14). Define the set

$$
D=\{i \kappa+j \sigma \mid i, j \in \mathbb{Z}\} .
$$

We observe, for later use, that if the ratio $\tan \theta=\sigma / \kappa$ is irrational, then $D$ is a dense subset of $\mathbb{R}$, while if $\tan \theta$ is either rational or infinite, then there exists a quantity $\nu>0$ such that

$$
D=\{n \nu \mid n \in \mathbb{Z}\},
$$

and so $D$ is a discrete subset of $\mathbb{R}$. We say that an element $u \in l^{\infty}\left(\mathbb{Z}^{2}\right)$ in the phase space of (1.1) is a monotone $\theta$-wave if there exists a function $\varphi: D \rightarrow \mathbb{R}$ such that

$$
u_{i, j}=\varphi(i \kappa+j \sigma) \text { for }(i, j) \in \mathbb{Z}^{2}
$$

and such that $\varphi$ is nonincreasing:

$$
\varphi\left(\xi_{1}\right) \leq \varphi\left(\xi_{2}\right) \text { if } \xi_{1} \leq \xi_{2} \text { with } \xi_{1}, \xi_{2} \in D
$$

Let $\mathbb{M}(\theta) \subseteq l^{\infty}\left(\mathbb{Z}^{2}\right)$ denote the set of all monotone $\theta$-waves $u$. The next result shows quite generally that the set $\mathbb{M}(\theta)$ is positively invariant for the system (1.1).

Theorem 2.1. Assume that $f: \mathbb{R} \rightarrow \mathbb{R}$ is locally Lipschitz and that $\alpha>0$, and consider a solution $u(t)$ of the lattice system (1.1). Suppose that $u(0) \in \mathbb{M}(\theta)$ for some $\theta \in \mathbb{R}$. Then $u(t) \in \mathbb{M}(\theta)$ for all $t \geq 0$ for which the solution is defined.

It is not hard to see that the unique solution of (1.1), with an initial condition $u(0) \in \mathbb{M}(\theta)$, is obtained by solving the system

$$
\varphi_{t}(t, \xi)=\alpha L \varphi(t, \xi)-f(\varphi(t, \xi)), \quad \xi \in D,
$$

with the appropriate initial condition and then setting $u_{i, j}(t)=\varphi(t, i \kappa+j \sigma)$. Here, $\varphi(t, \cdot) \in l^{\infty}(D)$ for each $t$, and $L: l^{\infty}(D) \rightarrow l^{\infty}(D)$ is the bounded linear operator given by

$$
L \varphi(\xi)=\varphi(\xi+\kappa)+\varphi(\xi-\kappa)+\varphi(\xi+\sigma)+\varphi(\xi-\sigma)-4 \varphi(\xi),
$$

so $(2.3)$ is an ODE in $l^{\infty}(D)$. To prove Theorem 2.1, we shall actually prove a slightly more abstract result for a class of systems containing (2.3). One easily sees that Theorem 2.1 is a direct consequence of the following result, via (2.3), with $\Lambda=$ $\alpha L+4 \alpha I$, and $g(u)=f(u)+4 \alpha u$.

Proposition 2.2. Let $D \subseteq \mathbb{R}$ be any countable set, let $\Lambda: l^{\infty}(D) \rightarrow l^{\infty}(D)$ be a bounded linear operator, and let $g: \mathbb{R} \rightarrow \mathbb{R}$ be a locally Lipschitz function. Define the set

$$
\mathbb{M}=\left\{\varphi \in l^{\infty}(D) \mid \varphi\left(\xi_{1}\right) \leq \varphi\left(\xi_{2}\right) \text { whenever } \xi_{1} \leq \xi_{2} \text {, with } \xi_{1}, \xi_{2} \in D\right\}
$$


and assume that

$$
\Lambda \varphi \in \mathbb{M} \text { whenever } \varphi \in \mathbb{M} \text {. }
$$

Consider the differential equation

$$
\frac{d \varphi}{d t}=\Lambda \varphi-g(\varphi)
$$

in the space $l^{\infty}(D)$, where by $g(\varphi)$ we mean coordinatewise evaluation, that is, $g(\varphi)(\xi)=$ $g(\varphi(\xi))$ for each $\xi \in D$ for $\varphi \in l^{\infty}(D)$. Then for any solution $\varphi(t, \xi)$ of $(2.5)$, we have that

$$
\varphi(t, \cdot) \in \mathbb{M} \text { for } t \geq 0 \text {, if } \varphi(0, \cdot) \in \mathbb{M} \text {, }
$$

for as long as this solution is defined.

Proof. First define a map $\rho: l^{\infty}(D) \rightarrow l^{\infty}(D)$ by

$$
\rho \varphi(\xi)=\sup _{\zeta \in(-\infty, \xi] \cap D} \varphi(\zeta) .
$$

Clearly, $\rho \varphi \in \mathbb{M}$, and also $\rho \varphi=\varphi$ holds whenever $\varphi \in \mathbb{M}$; that is, $\rho$ is a retraction of $l^{\infty}(D)$ onto $\mathbb{M}$. In addition, one easily checks that

$$
\left\|\rho \varphi_{1}-\rho \varphi_{2}\right\| \leq\left\|\varphi_{1}-\varphi_{2}\right\|,
$$

in the $l^{\infty}(D)$ norm, that is, $\rho$ is Lipschitz with Lipschitz constant 1.

Now consider, in place of (2.5), the system

$$
\frac{d \varphi}{d t}=\rho \Lambda \varphi-g(\varphi)
$$

with the same initial condition $\varphi(0, \cdot) \in \mathbb{M}$, and let $\widetilde{\varphi}(t, \xi)$ denote the solution of $(2.6)$ with this initial condition. Certainly, this solution is unique, as $\rho$ is Lipschitz. We shall prove that $\widetilde{\varphi}(t, \cdot) \in \mathbb{M}$ for all $t \geq 0$ for which this solution is defined. From this it follows that $\Lambda \widetilde{\varphi}(t, \cdot) \in \mathbb{M}$, hence that $\rho \Lambda \widetilde{\varphi}(t, \cdot)=\Lambda \widetilde{\varphi}(t, \cdot)$ for such $t$, since $\Lambda$ maps $\mathbb{M}$ into itself, and because $\rho$ is the identity on $\mathbb{M}$. Therefore, $\widetilde{\varphi}(t, \cdot)$ also satisfies (2.5), that is, $\varphi(t, \cdot)=\widetilde{\varphi}(t, \cdot) \in \mathbb{M}$, as desired.

To prove that $\widetilde{\varphi}(t, \cdot) \in \mathbb{M}$ for positive time, fix any two points $\xi_{1}, \xi_{2} \in D$, with $\xi_{1}<\xi_{2}$. We must prove that

$$
\widetilde{\varphi}\left(t, \xi_{1}\right) \leq \widetilde{\varphi}\left(t, \xi_{2}\right) \text { for } t \geq 0,
$$

starting at $t=0$ where $(2.7)$ holds. Let $\eta(t)=\widetilde{\varphi}\left(t, \xi_{2}\right)-\widetilde{\varphi}\left(t, \xi_{1}\right)$. Then for any compact interval $[0, T]$ on which the solution is defined, there exists a constant $K$, such that

$$
\begin{aligned}
\dot{\eta}(t) & =\rho \Lambda \widetilde{\varphi}\left(t, \xi_{2}\right)-\rho \Lambda \widetilde{\varphi}\left(t, \xi_{1}\right)-g\left(\widetilde{\varphi}\left(t, \xi_{2}\right)\right)+g\left(\widetilde{\varphi}\left(t, \xi_{1}\right)\right) \\
& \geq-g\left(\widetilde{\varphi}\left(t, \xi_{2}\right)\right)+g\left(\widetilde{\varphi}\left(t, \xi_{1}\right)\right) \\
& \geq-K\left|\widetilde{\varphi}\left(t, \xi_{2}\right)-\widetilde{\varphi}\left(t, \xi_{1}\right)\right|=-K|\eta(t)|
\end{aligned}
$$

where in the first inequality we have used the fact that $\rho \Lambda \widetilde{\varphi}(t, \cdot) \in \mathbb{M}$, and where $K$ is an appropriate local Lipschitz constant for $g$. But now, because $\eta(0) \geq 0$, it follows immediately from the differential inequality $(2.8)$ that $\eta(t) \geq e^{-K t} \eta(0)$ for $t \geq 0$. This establishes (2.7), and proves the proposition.

Remark. A general open question, suggested by the above result, is to determine the ultimate evolution of solutions of the system (1.1) which are monotone $\theta$-waves. In particular, one can ask when such solutions approach traveling waves (1.15) in the class $\mathbb{M}(\theta)$. 
3. Discontinuous nonlinearities. It is generally very difficult to obtain rigorous results of a global nature, beyond the most elementary properties, for systems such as (1.1) with a general nonlinearity $f$. One approach, which has the potential to yield some understanding of the differential equation, is to consider idealized nonlinearities $f$ which are either step functions, or are piecewise linear. In such cases, one hopes that the system (1.1) will succumb to explicit calculations which can provide insight into the dynamics of the problem. Indeed, McKean [28] adopted this approach in his studies of the Nagumo equation.

In taking this approach, there arises the issue of whether such special nonlinearities are representative of more general $f$, that is, whether the phenomena observed persist in a robust fashion for a larger class of $f$, or whether they are merely artifacts of the discontinuities in $f$ and its derivative $f^{\prime}$. One must be prepared to prove theorems which address such issues, as they arise in the course of the calculations. We mention that this approach, of studying idealized nonlinearities, has been taken in [26] with some success for classes of differential-delay equations arising in a different context, and we shall see that it bears fruit in the present case.

Below, we consider a class of piecewise linear $f$ which are modeled on the cubic (1.3). Specifically, we consider

$$
f(u)=f(u, a)=u-h(u-a),
$$

where $h$ denotes the Heaviside function

$$
h(u)=\left\{\begin{array}{cc}
0, & u<0, \\
1, & u>0,
\end{array}\right.
$$

and where $a \in(0,1)$ as before. Our interest is in obtaining two-dimensional traveling wave solutions (1.15) of (1.1), specifically, solutions (1.16) satisfying the boundary conditions (1.11). In particular, we wish to study the dependence of these solutions on the parameters $\alpha$ and $a$ in the system, and also on the direction of propagation $e^{i \theta}$ of the wave.

The issue immediately arises as to what one means by a solution of (1.1) when $f$ is discontinuous. For our purposes, it will be sufficient to regard $f$ (and the Heaviside function $h$ ) as set-valued functions, with $f(u)$ and $h(u)$ singleton sets for $u \neq a$, and $u \neq 0$, respectively, and

$$
f(a)=[a-1, a], \quad h(0)=[0,1],
$$

compact intervals given by "filling in" the jump discontinuities in the graphs of $f$ and $h$. In this setting, by a solution of (1.1) we mean $u(t) \in l^{\infty}\left(\mathbb{Z}^{2}\right)$ for which each coordinate function $u_{i, j}(t)$ is absolutely continuous, and satisfies the differential inclusion

$$
\dot{u}_{i, j}(t) \in \alpha(\Delta u(t))_{i, j}-f\left(u_{i, j}(t)\right)
$$

for almost every $t$. One can make a corresponding characterization of a solution of the differential-difference equation (1.16), at least when $c \neq 0$. In fact, each solution of (1.16) for $c \neq 0$ and with $f$ as in (3.1) that we obtain, will satisfy $\varphi(\xi)=a$ at only one value of $\xi$. At that point, the derivative $\varphi^{\prime}(\xi)$ with respect to $\xi$ will have a jump discontinuity, and elsewhere the solution will be $C^{1}$ and will satisfy (1.16) in the classical sense. 
4. Nonzero wave speed. In this section we fix the piecewise linear function $f$ as in (3.1), (3.2), with $a \in(0,1)$, and consider solutions of (1.16) for some $c \neq 0$, with the boundary conditions (1.11). As always, $\sigma$ and $\kappa$ are given by (1.14). We keep $\alpha>0$ fixed.

In what follows, for ease of notation we shall let $L \varphi$ denote the expression (2.4). We do not necessarily assume that $L$ is to be interpreted as an operator on the space $l^{\infty}(D)$, as before, although we shall say so when this is the case. Generally, we shall allow the variable $\xi$ in the expression $L \varphi(\xi)$ to take any real value.

We shall construct solutions of (1.16), (1.11) using the Fourier transform. However, a certain amount of care is needed in order that one may rigorously justify the calculations. In particular, we shall initially assume that our solution $\varphi$ satisfies $\varphi(\xi)=a$ for only one value of $\xi$. Without loss we may take this value to be $\xi=0$ (by translating the argument $\xi$ ), and therefore, we will assume that

$$
\varphi(\xi)<a \text { for } \xi<0, \quad \varphi(\xi)>a \text { for } \xi>0,
$$

in view of the boundary conditions (1.11). We are thus excluding any solutions which cross the value $\varphi(\xi)=a$ more than once. It follows from (4.1) that one has

$$
h(\varphi(\xi)-a)=h(\xi) \text { for } \xi \neq 0,
$$

and so $f(\varphi(\xi))=\varphi(\xi)-h(\xi)$ for $\xi \neq 0$, by (3.1). With the condition (4.2), the system (1.16) now takes the form

$$
-c \varphi^{\prime}(\xi)=\alpha L \varphi(\xi)-\varphi(\xi)+h(\xi)
$$

of a linear inhomogeneous equation which can be approached with transform methods. The assumption (4.1) on the solution $\varphi$ will be justified a posteriori; that is, after we construct $\varphi$ satisfying (4.3), and also $\varphi(0)=a$, we shall show that it also satisfies (4.1), and so is indeed a solution of (1.16).

We prove, in Theorem 4.6, that the solution $\varphi$ is in fact strictly increasing throughout all of $\mathbb{R}$. One expects that every solution of (1.16), (1.11) that is increasing for all large $|\xi|$ is increasing on $\mathbb{R}$. While we do not actually prove this fact for the nonlinearity (3.1) (although see [23], however), we do see that it holds for a class of continuous $f$, including the cubic (1.3), in a remark following the proof of Theorem 4.6.

The Fourier transform of $\varphi$, given by

$$
\widehat{\varphi}(s)=\int_{-\infty}^{\infty} e^{-i s \xi} \varphi(\xi) d \xi,
$$

is well defined for complex $s$ satisfying $\operatorname{Im} s<0$, with $|\operatorname{Im} s|$ sufficiently small, provided that $\varphi(\xi)$ is bounded as $\xi \rightarrow \infty$, and that $\varphi(\xi) \rightarrow 0$ exponentially fast as $\xi \rightarrow-\infty$. (Of course, with the boundary conditions (1.11) there is a problem with the convergence of (4.4) for $\operatorname{Im} s \geq 0$.) Before proceeding further, we need the following lemma as justification of our calculations.

Lemma 4.1. Let $\varphi$ be a solution of (4.3), (1.11) for some $c \neq 0$. Then there exists $\varepsilon_{0}>0$ such that

$$
|\varphi(\xi)| \leq K e^{\varepsilon_{0} \xi} \text { for } \xi \leq 0
$$

for some $K>0$. 
Proof. Let $\psi(\xi)=\varphi(-\xi)$. Then from (4.3) we have that

$$
c \psi^{\prime}(\xi)=\alpha L \psi(\xi)-\psi(\xi)
$$

for all $\xi>0$, with $\psi(\xi) \rightarrow 0$ as $\xi \rightarrow \infty$, by (1.11). Let $\widehat{\psi}(\xi)$ denote (for this proof only) the Laplace transform

$$
\widehat{\psi}(s)=\int_{0}^{\infty} e^{-s \xi} \psi(\xi) d \xi
$$

which is analytic in the right half-plane $\operatorname{Re} s>0$. In a standard fashion, after a calculation, we obtain from (4.6) that

$$
\widehat{\psi}(s)=\frac{q(s)}{P(s)},
$$

where

$$
\begin{gathered}
q(s)=c \psi(0)-\alpha\left(e^{\kappa s} \int_{0}^{\kappa} e^{-s \xi} \psi(\xi) d \xi-e^{-\kappa s} \int_{-\kappa}^{0} e^{-s \xi} \psi(\xi) d \xi\right. \\
\left.+e^{\sigma s} \int_{0}^{\sigma} e^{-s \xi} \psi(\xi) d \xi-e^{-\sigma s} \int_{-\sigma}^{0} e^{-s \xi} \psi(\xi) d \xi\right), \\
P(s)=c s+1-\alpha\left(e^{\kappa s}+e^{\sigma s}+e^{-\kappa s}+e^{-\sigma s}-4\right) .
\end{gathered}
$$

By integrating by parts, we see that

$$
\int_{0}^{\kappa} e^{-s \xi} \psi(\xi) d \xi=-\left.\frac{e^{-s \xi} \psi(\xi)}{s}\right|_{\xi=0} ^{\kappa}+\frac{1}{s} \int_{0}^{\kappa} e^{-s \xi} \psi^{\prime}(\xi) d \xi
$$

and similarly for the other three integrals in (4.8). In particular, one has that $q(s)=$ $c \psi(0)+O\left(|s|^{-1}\right)$ as $|\operatorname{Im} s| \rightarrow \infty$, uniformly for Re $s$ bounded. From this and also from the formula for $P(s)$, therefore, one easily has from (4.7) that

$$
\widehat{\psi}(s)=\frac{\psi(0)}{s}+O\left(|s|^{-2}\right) \text { as }|\operatorname{Im} s| \rightarrow \infty,
$$

uniformly for Re $s$ bounded. But now the asymptotic expression (4.9) justifies the inversion formula for the Laplace transform, and in particular justifies shifting the integration contour around any singularities on the imaginary axis. We therefore have

$$
\begin{aligned}
\psi(\xi) & =\frac{1}{2 \pi i} \int_{\varepsilon_{0}-i \infty}^{\varepsilon_{0}+i \infty} e^{s \xi} \widehat{\psi}(s) d s \\
& =\frac{1}{2 \pi i} \int_{-\varepsilon_{0}-i \infty}^{-\varepsilon_{0}+i \infty} e^{s \xi} \widehat{\psi}(s) d s+\frac{1}{2 \pi i} \sum_{\operatorname{Re} s=0} e^{s \xi} \operatorname{res}(\widehat{\psi}, s) \\
& =\frac{1}{2 \pi i} \int_{-\varepsilon_{0}-i \infty}^{-\varepsilon_{0}+i \infty} e^{s \xi}\left(\widehat{\psi}(s)-\frac{\psi(0)}{s}\right) d s+\frac{1}{2 \pi i} \sum_{\operatorname{Re} s=0} e^{s \xi} \operatorname{res}(\widehat{\psi}, s),
\end{aligned}
$$


where $\varepsilon_{0}>0$ is sufficiently small, where, generally, $\operatorname{res}(g, z)$ denotes the residue of a meromorphic function $g$ at a point $z$, and where the sum in (4.10) is taken over all poles of $\widehat{\psi}$ on the imaginary axis, if any exist. The limits of integration denote contour integrals along the vertical lines $t \rightarrow \pm \varepsilon_{0}+i t$ for $t \in \mathbb{R}$ in the obvious way.

The first equality in (4.10) is simply the Laplace inversion formula, and is justified by the fact that $\widehat{\psi}$ has no poles in the right half plane. The second equality in (4.10) follows from shifting the contour of integration (in this case, a vertical line) around any poles of $\widehat{\psi}$ on the imaginary axis, and $\varepsilon_{0}>0$ is small enough that $\widehat{\psi}$ has no poles in the strip $-\varepsilon_{0} \leq \operatorname{Re} s<0$. The third equality in (4.10) follows from the identity

$$
\frac{1}{2 \pi i} \int_{\varepsilon-i \infty}^{\varepsilon+i \infty} \frac{e^{s \xi}}{s} d s=\left\{\begin{array}{cc}
1, & \varepsilon>0, \\
0, & \varepsilon<0,
\end{array}\right.
$$

for $\xi>0$, which is just the inversion formula for the function $g(\xi)=1$ and its transform $\widehat{g}(s)=1 / s$.

It is now clear from (4.9) that the third integral in (4.10) enjoys the estimate

$$
\left|\frac{1}{2 \pi i} \int_{-\varepsilon_{0}-i \infty}^{-\varepsilon_{0}+i \infty} e^{s \xi}\left(\widehat{\psi}(s)-\frac{\psi(0)}{s}\right) d s\right| \leq K e^{-\varepsilon_{0} \xi} \text { for } \xi \geq 0
$$

for some $K>0$; in particular, this term approaches zero as $\xi \rightarrow \infty$. By assumption, we also have that $\psi(\xi) \rightarrow 0$ as $\xi \rightarrow \infty$, and it follows directly from this that each of the residues in the summation in (4.10) vanishes, that is,

$$
\operatorname{res}(\widehat{\psi}, s)=0 \text { for } \operatorname{Im} s=0 .
$$

Thus, $|\psi(\xi)| \leq K e^{-\varepsilon_{0} \xi}$ for $\xi \geq 0$, which is equivalent to (4.5), as desired.

Let us now proceed with the construction of a solution to (4.3), (1.11). In order to justify our calculations more properly, let us consider the Fourier transform

$$
\widehat{\varphi}_{\varepsilon}(s)=\int_{-\infty}^{\infty} e^{-i s \xi} \varphi_{\varepsilon}(\xi) d \xi, \quad \varphi_{\varepsilon}(\xi)=e^{-\varepsilon \xi} \varphi(\xi)
$$

where $\varepsilon>0$ is sufficiently small. By Lemma 4.1, if $\varphi$ satisfies (4.3), (1.11), then $\varphi_{\varepsilon}(\xi) \rightarrow 0$ exponentially fast, both as $\xi \rightarrow-\infty$ and $\xi \rightarrow \infty$, provided that $0<\varepsilon<\varepsilon_{0}$. In particular, $\varphi_{\varepsilon}$ belongs to $L^{1}(\mathbb{R}) \cap L^{2}(\mathbb{R})$ in this case. Also, from (4.3), the function $\varphi_{\varepsilon}(\xi)$ satisfies the equation

$$
\begin{aligned}
-c \varphi_{\varepsilon}^{\prime}(\xi)=\alpha\left(e^{\varepsilon \kappa} \varphi_{\varepsilon}(\xi+\kappa)+e^{-\varepsilon \kappa} \varphi_{\varepsilon}(\xi-\kappa)+e^{\varepsilon \sigma} \varphi_{\varepsilon}(\xi+\sigma)\right. & \\
& \left.+e^{-\varepsilon \sigma} \varphi_{\varepsilon}(\xi-\sigma)-4 \varphi_{\varepsilon}(\xi)\right)-(1-c \varepsilon) \varphi_{\varepsilon}(\xi)+e^{-\varepsilon \xi} h(\xi) .
\end{aligned}
$$

Taking the Fourier transform of both sides of (4.11) yields, in a standard fashion,

$$
\widehat{\varphi}_{\varepsilon}(s)=\frac{1}{(i s+\varepsilon) R(s-i \varepsilon)},
$$

where

$$
\begin{aligned}
R(s) & =-i c s+1-\alpha\left(e^{i \kappa s}+e^{-i \kappa s}+e^{i \sigma s}+e^{-i \sigma s}-4\right) \\
& =-i c s+1+2 \alpha(2-\cos \kappa s-\cos \sigma s)
\end{aligned}
$$


Clearly, $\widehat{\varphi}_{\varepsilon} \in L^{1}(\mathbb{R}) \cap L^{2}(\mathbb{R})$, and so by the Fourier inversion theorem,

$$
\varphi(\xi)=e^{\varepsilon \xi} \varphi_{\varepsilon}(\xi)=\frac{1}{2 \pi} \int_{-\infty}^{\infty} e^{(i s+\varepsilon) \xi} \widehat{\varphi}_{\varepsilon}(s) d s,
$$

which is an absolutely convergent integral. Using (4.12), we write (4.14) as

$$
\varphi(\xi)=\frac{1}{2 \pi i} \int_{-i \varepsilon-\infty}^{-i \varepsilon+\infty} \frac{e^{i s \xi}}{s R(s)} d s
$$

where again the limits of integration denote the obvious contour integral along a horizontal line. We note that, apart from a simple pole at $s=0$, the integrand in (4.15) is holomorphic in the horizontal strip $-\varepsilon \leq \operatorname{Im} s \leq 0$ for small $\varepsilon$. We therefore wish to shift the contour of integration in (4.15) to obtain

$$
\varphi(\xi)=\frac{1}{2 \pi i}\left(\int_{\mathcal{C}_{\varepsilon}}+\int_{\mathcal{S}_{\varepsilon}}\right) \frac{e^{i s \xi}}{s R(s)} d s,
$$

where $\mathcal{C}_{\varepsilon}$ denotes the two half-lines $(-\infty,-\varepsilon]$ and $[\varepsilon, \infty)$ in the real axis, and $\mathcal{S}_{\varepsilon}$ denotes the half-circle $t \rightarrow \varepsilon e^{i t}$ for $-\pi \leq t \leq 0$, with the contour oriented in the direction of increasing $\operatorname{Re} s$. For any fixed $\xi \geq 0$, such a shift of the contour is justified by the boundedness of $e^{i s \xi}$ in the strip $-\varepsilon \leq \operatorname{Im} s \leq 0$, and by the uniform estimate $1 /(s R(s))=O\left(|s|^{-2}\right)$ of decay as $|\operatorname{Re} s| \rightarrow \infty$ in this strip.

The integrals along the two components of $\mathcal{C}_{\varepsilon}$ may be combined, giving

$$
\begin{aligned}
\frac{1}{2 \pi i} \int_{\mathcal{C}_{\varepsilon}} \frac{e^{i s \xi}}{s R(s)} d s & =\frac{1}{2 \pi i}\left(\int_{-\infty}^{-\varepsilon}+\int_{\varepsilon}^{\infty}\right) \frac{e^{i s \xi}}{s R(s)} d s \\
& =\frac{1}{2 \pi i} \int_{\varepsilon}^{\infty} \frac{e^{i s \xi}}{s R(s)}-\frac{e^{-i s \xi}}{s R(-s)} d s \\
& =\frac{1}{\pi} \int_{\varepsilon}^{\infty} \frac{A(s) \sin s \xi}{s\left(A(s)^{2}+c^{2} s^{2}\right)} d s+\frac{c}{\pi} \int_{\varepsilon}^{\infty} \frac{\cos s \xi}{A(s)^{2}+c^{2} s^{2}} d s
\end{aligned}
$$

where we denote

$$
A(s)=1+2 \alpha(2-\cos \kappa s-\cos \sigma s)
$$

as the real part of $R(s)$, when $s$ is real. The integral around the half-circle $\mathcal{S}_{\varepsilon}$ yields $2 \pi i$ times half the residue of the integrand at $s=0$, as $\varepsilon \rightarrow 0$; that is,

$$
\frac{1}{2 \pi i} \int_{\mathcal{S}_{\varepsilon}} \frac{e^{i s \xi}}{s R(s)} d s=\frac{1}{2}+o(1) \text { as } \varepsilon \rightarrow 0 .
$$

Inserting (4.17) and (4.19) into (4.16) and letting $\varepsilon \rightarrow 0$, we obtain

$$
\varphi(\xi)=\frac{1}{2}+\frac{1}{\pi} \int_{0}^{\infty} \frac{A(s) \sin s \xi}{s\left(A(s)^{2}+c^{2} s^{2}\right)} d s+\frac{c}{\pi} \int_{0}^{\infty} \frac{\cos s \xi}{A(s)^{2}+c^{2} s^{2}} d s,
$$

an explicit formula for our solution.

Remark. The formula (4.20) was derived under the assumption that $\varphi$ was a solution of equation (4.3), for some $c \neq 0$, and satisfied the boundary conditions (1.11). 
Conversely, by construction, the function $\varphi$ given by (4.20) satisfies the differential equation (4.3).

Remark. From (4.20), we see directly the symmetry property

$$
\varphi(\xi, c)=1-\varphi(-\xi,-c)
$$

for the solution $\varphi(\xi)=\varphi(\xi, c)$.

Remark. From (4.15), we have that $|\varphi(\xi)|=O\left(e^{\varepsilon \xi}\right)$ as $\xi \rightarrow-\infty$ and so $\varphi$ satisfies the first boundary condition $\varphi(-\infty)=0$ in (1.11). By the symmetry property (4.21), the other boundary condition $\varphi(\infty)=1$ of (1.11) also holds.

The following result describes additional properties of $\varphi$, which will be needed shortly.

Proposition 4.2. Letting $\varphi(\xi, c)$ denote the function in (4.20), we have that $\varphi(\xi, c)$ is analytic in $c$ and jointly continuous in $(\xi, c)$ for real $c \neq 0$. In addition, for each such $c$, the derivative

$$
\psi(\xi)=\psi(\xi, c)=D_{c} \varphi(\xi, c)
$$

with respect to $c$ is bounded as $\xi \rightarrow \pm \infty$ and for $\xi \neq 0$ satisfies the equation

$$
-\varphi^{\prime}(\xi)-c \psi^{\prime}(\xi)=\alpha L \psi(\xi)-\psi(\xi),
$$

obtained by differentiating (4.3) with respect to $c$.

Proof. We shall actually prove the claims about $\varphi$ and its derivative for all $c \in \mathbb{C}$ with $\operatorname{Re} c \neq 0$. For any compact subset of $\{c \in \mathbb{C} \mid \operatorname{Re} c \neq 0\}$, there exists a uniform lower bound

$$
\left|A(s)^{2}+c^{2} s^{2}\right| \geq K_{1}\left(1+s^{2}\right) \text { for } s \geq 0
$$

for the term in the denominators of (4.20). (Note that if $\operatorname{Re} c=0$ and $c \neq 0$, then $A(s)^{2}+c^{2} s^{2}=0$ at some $s>0$.) This in turn yields a uniform upper bound of the form $K_{2}\left(1+s^{2}\right)^{-1}$, valid for bounded $\xi$, for each of the two integrands in (4.20). This estimate, and the analyticity of the integrands in $c$ and continuity in $(\xi, c)$ for each $s$, imply the analyticity and continuity claims for $\varphi$.

The boundedness of $\psi$ is easily proved by differentiating the formula (4.20) with respect to $c$ under the integral sign, again using the estimate (4.23). Differentiating (4.3) yields (4.22), once one justifies exchanging the order of derivatives, $\psi^{\prime}(\xi, c)=$ $D_{c} \varphi^{\prime}(\xi, c)$. However, this follows from standard arguments.

In order that $\varphi$ satisfy (1.16) with $f$ as in (3.1), it is necessary that

$$
\varphi(\xi) \leq a \text { for } \xi<0, \quad \varphi(\xi) \geq a \text { for } \xi>0
$$

both hold. In particular, we require that $\varphi(0)=a$, or equivalently that

$$
a-\frac{1}{2}=\frac{c}{\pi} \int_{0}^{\infty} \frac{d s}{A(s)^{2}+c^{2} s^{2}}
$$

We see that this formula expresses the relation between the parameter $a$ in the nonlinearity, and the wave speed $c$ of the solution. Later, we shall prove that in fact the strict inequalities (4.1) hold whenever (4.25) holds, with $c \neq 0$.

Let us define the function

$$
\Gamma(c)=\varphi(0, c)-\frac{1}{2}=\frac{c}{\pi} \int_{0}^{\infty} \frac{d s}{A(s)^{2}+c^{2} s^{2}},
$$


the right-hand side of (4.25). Clearly, $\Gamma$ is an odd function of $c$, and by Proposition 4.2, it is analytic for (real) $c \neq 0$, where the integral (4.26) converges. On the other hand, $\Gamma$ is discontinuous at $c=0$, as the following result shows. Indeed, the right-hand limit $\Gamma(0+)$ is not zero, but rather is a positive quantity $\Gamma(0+)=\gamma$.

TheOREM 4.3. Let $\Gamma(c)$ be defined by (4.26) for each real $c \neq 0$. Then $\Gamma$ is odd and analytic, and

$$
|\Gamma(c)|<\frac{1}{2}
$$

for all such $c$. In addition,

$$
\lim _{c \rightarrow \infty} \Gamma(c)=\frac{1}{2}, \quad \lim _{c \rightarrow 0+} \Gamma(c)=\gamma,
$$

where $\gamma>0$ is the quantity

$$
\gamma=\lim _{T \rightarrow \infty} \frac{1}{2 T} \int_{0}^{T} \frac{d s}{A(s)}
$$

where the limit in (4.29) always exists.

Proof. The oddness of $\Gamma$ is obvious, and the analyticity follows from Proposition 4.2. The inequality (4.27) follows from the fact that $A(s) \geq 1$, with strict inequality for some $s$, which gives

$$
|\Gamma(c)|<\frac{|c|}{\pi} \int_{0}^{\infty} \frac{d s}{1+c^{2} s^{2}}=\frac{1}{2} .
$$

To prove the limits (4.28), change variables in (4.26) to obtain (with $c>0$ )

$$
\Gamma(c)=\frac{1}{\pi} \int_{0}^{\infty} \frac{d s}{A(s / c)^{2}+s^{2}} .
$$

An application of the Lebesgue dominated convergence theorem, and the observation that $A(0)=1$, yield the first limit in (4.28).

The second limit, $c \rightarrow 0+$, on the other hand, is more subtle. Let us first observe that the function $A$ is almost periodic. By a basic property of almost periodic functions (see, for example, [36]), given any $\varepsilon>0$ there exists $\tau_{\varepsilon}>0$ such that every interval of length $\tau_{\varepsilon}$ contains an $\varepsilon$-translation number $T$, that is, a number $T$ for which $|A(s+T)-A(s)| \leq \varepsilon$ for all $s \in \mathbb{R}$. Moreover, we may assume without loss that $\tau_{\varepsilon} \rightarrow \infty$ as $\varepsilon \rightarrow 0$. There exists in particular an $\varepsilon$-translation number $T_{\varepsilon}$ satisfying $T_{\varepsilon} \in\left[\tau_{\varepsilon}, 2 \tau_{\varepsilon}\right]$. Let $\varepsilon$, and the quantities $\tau_{\varepsilon}$ and $T_{\varepsilon}$, remain fixed in the following argument until we note otherwise. We shall assume that $\varepsilon \leq 1 / 4$.

Define a function $A_{\varepsilon}: \mathbb{R} \rightarrow \mathbb{R}$ of period $T_{\varepsilon}$ by setting

$$
A_{\varepsilon}(s)=A(s) \text { for } 0 \leq s<T_{\varepsilon}, \quad A_{\varepsilon}\left(s+T_{\varepsilon}\right)=A_{\varepsilon}(s) \text { for } s \in \mathbb{R} .
$$

We claim the following: that for every integer $n$, there exists $\rho_{n} \in \mathbb{R}$ such that

$$
\left|A(s)-A_{\varepsilon}\left(s+\rho_{n}\right)\right| \leq 2 \varepsilon \text { for } n T_{\varepsilon} \leq s<(n+1) T_{\varepsilon} .
$$

To prove this claim, let us first observe that

$$
\left|A(s)-A_{\varepsilon}(s)\right| \leq \varepsilon \text { for }-T_{\varepsilon} \leq s<T_{\varepsilon} .
$$


Indeed, the inequality in (4.31) is trivial for $0 \leq s<T_{\varepsilon}$, and for $-T_{\varepsilon} \leq s<0$ we have that

$$
\left|A(s)-A_{\varepsilon}(s)\right|=\left|A(s)-A_{\varepsilon}\left(s+T_{\varepsilon}\right)\right|=\left|A(s)-A\left(s+T_{\varepsilon}\right)\right| \leq \varepsilon,
$$

by the periodicity of $A_{\varepsilon}$ and the fact that $T_{\varepsilon}$ is an $\varepsilon$-translation number for $A$. Now fix any integer $n$, and let $\rho_{n}$ be an $\varepsilon$-translation number for $A$ satisfying $\rho_{n} \in[-(n+$ 1) $T_{\varepsilon},-n T_{\varepsilon}$ ]. This interval contains such $\rho_{n}$, as its length is $T_{\varepsilon} \geq \tau_{\varepsilon}$. For any $s$ satisfying $n T_{\varepsilon} \leq s<(n+1) T_{\varepsilon}$ we have that $-T_{\varepsilon} \leq s+\rho_{n}<T_{\varepsilon}$, and hence that

$$
\left|A(s)-A_{\varepsilon}\left(s+\rho_{n}\right)\right| \leq\left|A(s)-A\left(s+\rho_{n}\right)\right|+\left|A\left(s+\rho_{n}\right)-A_{\varepsilon}\left(s+\rho_{n}\right)\right| \leq \varepsilon+\varepsilon=2 \varepsilon,
$$

where we use the fact that $\rho_{n}$ is an $\varepsilon$-translation number; we also have (4.31). This establishes the claim.

For ease of notation let us denote $A_{\varepsilon}^{n}(s)=A_{\varepsilon}\left(s+\rho_{n}\right)$ for every $n$. Noting by (4.18) that $1 \leq A(s) \leq 1+8 \alpha$, we have that

$$
\left|A(s)+A_{\varepsilon}^{n}(s)\right| \leq 2 A(s)+2 \varepsilon \leq 2+16 \alpha+2 \varepsilon
$$

for $n T_{\varepsilon} \leq s<(n+1) T_{\varepsilon}$. Writing the integrand in (4.30) as a sum of integrals from $n c T_{\varepsilon}$ to $(n+1) c T_{\varepsilon}$ for $n \geq 0$, we have that

$$
\begin{aligned}
\mid \Gamma(c)- & \frac{1}{\pi} \sum_{n=0}^{\infty} \int_{n c T_{\varepsilon}}^{(n+1) c T_{\varepsilon}} \frac{d s}{A_{\varepsilon}^{n}(s / c)^{2}+s^{2}} \mid \\
& =\frac{1}{\pi}\left|\sum_{n=0}^{\infty} \int_{n c T_{\varepsilon}}^{(n+1) c T_{\varepsilon}}\left(\frac{1}{\left(A(s / c)^{2}+s^{2}\right)}-\frac{1}{\left(A_{\varepsilon}^{n}(s / c)^{2}+s^{2}\right)}\right) d s\right| \\
& =\frac{1}{\pi}\left|\sum_{n=0}^{\infty} \int_{n c T_{\varepsilon}}^{(n+1) c T_{\varepsilon}} \frac{A_{\varepsilon}^{n}(s / c)^{2}-A(s / c)^{2}}{\left(A(s / c)^{2}+s^{2}\right)\left(A_{\varepsilon}^{n}(s / c)^{2}+s^{2}\right)} d s\right| \\
& \leq \frac{1}{\pi} \int_{0}^{\infty} \frac{(2+16 \alpha+2 \varepsilon) 2 \varepsilon}{\left(1+s^{2}\right)\left((1-2 \varepsilon)^{2}+s^{2}\right)} d s \leq\left(\frac{2+16 \alpha+2 \varepsilon}{(1-2 \varepsilon)^{2}}\right) \varepsilon \leq(10+64 \alpha) \varepsilon
\end{aligned}
$$

where we have used the fact that $A_{\varepsilon}^{n}(s / c) \geq 1-2 \varepsilon \geq 1 / 2$. We have further, using the periodicity of $A_{\varepsilon}$, that

$$
\begin{aligned}
\frac{1}{\pi} \sum_{n=0}^{\infty} \int_{n c T_{\varepsilon}}^{(n+1) c T_{\varepsilon}} \frac{d s}{A_{\varepsilon}^{n}(s / c)^{2}+s^{2}} & =\frac{1}{\pi} \sum_{n=0}^{\infty}\left(\int_{n c T_{\varepsilon}}^{(n+1) c T_{\varepsilon}} \frac{d s}{A_{\varepsilon}^{n}(s / c)^{2}+\left(n c T_{\varepsilon}\right)^{2}}+E_{n}\right) . \\
& =\frac{1}{\pi} \sum_{n=0}^{\infty}\left(c \int_{0}^{T_{\varepsilon}} \frac{d s}{A_{\varepsilon}(s)^{2}+\left(n c T_{\varepsilon}\right)^{2}}+E_{n}\right),
\end{aligned}
$$

where the error term

$$
E_{n}=\int_{n c T_{\varepsilon}}^{(n+1) c T_{\varepsilon}} \frac{\left(n c T_{\varepsilon}\right)^{2}-s^{2}}{\left(A_{\varepsilon}^{n}(s / c)^{2}+s^{2}\right)\left(A_{\varepsilon}^{n}(s / c)^{2}+\left(n c T_{\varepsilon}\right)^{2}\right)} d s
$$

is easily seen to satisfy

$$
\left|E_{n}\right| \leq \int_{n c T_{\varepsilon}}^{(n+1) c T_{\varepsilon}} \frac{(2 n+1)\left(c T_{\varepsilon}\right)^{2}}{\left(1 / 4+s^{2}\right)\left(1 / 4+s^{2} / 4\right)} d s \leq 48 c T_{\varepsilon} \int_{n c T_{\varepsilon}}^{(n+1) c T_{\varepsilon}} \frac{s}{\left(1+s^{2}\right)^{2}} d s
$$


for $n \neq 0$, where we have again used the fact that $A_{\varepsilon}(s / c) \geq 1 / 2$. Also, $\left|E_{0}\right| \leq$ $16\left(c T_{\varepsilon}\right)^{3} / 3$ is easily verified. At this point, note from (4.34) that

$$
\sum_{n=0}^{\infty}\left|E_{n}\right| \leq 16\left(c T_{\varepsilon}\right)^{3} / 3+48 c T_{\varepsilon} \int_{c T_{\varepsilon}}^{\infty} \frac{s}{\left(1+s^{2}\right)^{2}} d s \rightarrow 0 \text { as } c \rightarrow 0 .
$$

Denoting

$$
F(t)=\frac{1}{T_{\varepsilon}} \int_{0}^{T_{\varepsilon}} \frac{d s}{A_{\varepsilon}(s)^{2}+t^{2}}=\frac{1}{T_{\varepsilon}} \int_{0}^{T_{\varepsilon}} \frac{d s}{A(s)^{2}+t^{2}},
$$

we may write the final summation involving the integral in (4.33) as

$$
\frac{1}{\pi} \sum_{n=0}^{\infty} c \int_{0}^{T_{\varepsilon}} \frac{d s}{A_{\varepsilon}(s)^{2}+\left(n c T_{\varepsilon}\right)^{2}} d s=\frac{c T_{\varepsilon}}{\pi} \sum_{n=0}^{\infty} F\left(n c T_{\varepsilon}\right)
$$

Regarding the right-hand side of (4.36) as a Riemann sum, and noting the limit (4.35), we have from (4.33) that

$$
\begin{aligned}
\lim _{c \rightarrow 0} \frac{1}{\pi} \sum_{n=0}^{\infty} \int_{n c T_{\varepsilon}}^{(n+1) c T_{\varepsilon}} \frac{d s}{A_{\varepsilon}^{n}(s / c)^{2}+s^{2}}=\frac{1}{\pi} \int_{0}^{\infty} F(t) d t \\
=\frac{1}{\pi T_{\varepsilon}} \int_{0}^{T_{\varepsilon}} \int_{0}^{\infty} \frac{1}{A(s)^{2}+t^{2}} d t d s=\frac{1}{2 T_{\varepsilon}} \int_{0}^{T_{\varepsilon}} \frac{d s}{A(s)},
\end{aligned}
$$

where an application of Fubini's theorem has allowed us to interchange the order of integration in (4.37). To complete the proof of the theorem, we simply note that the second limit in (4.28), with $\gamma$ as in (4.29), follows directly from (4.32) and (4.37) by letting $\varepsilon \rightarrow 0$.

Remark. Let us observe from (4.18) and (4.29), that $\gamma \rightarrow 1 / 2$ as $\alpha \rightarrow 0$. This is consistent with our earlier observations for smooth $f$, showing that for small coupling parameter $\alpha$, the interval $[1 / 2-\gamma, 1 / 2+\gamma]$ of propagation failure expands throughout the interval $(0,1)$.

Remark. With a bit more effort, we see that $\gamma \rightarrow 0$ as $\alpha \rightarrow \infty$. As the limit $\alpha \rightarrow \infty$ corresponds to the continuous PDE limit (1.6) of the discrete equation (1.1), this is consistent with the fact that there is no propagation failure for the PDE. To prove that $\gamma \rightarrow 0$ for this limit, take any positive $\delta<1$, let $\cos \varepsilon=1-\delta$, with $\varepsilon \in(0, \pi / 2)$, and define

$$
J_{n}=[(2 \pi n-\varepsilon) /|\kappa|,(2 \pi n+\varepsilon) /|\kappa|], \quad J=\bigcup_{n=-\infty}^{\infty} J_{n},
$$

assuming that $\kappa \neq 0$ (if $\kappa=0$, take $\sigma= \pm 1$ in place of $\kappa$ ). By (4.18), if $A(s) \leq 1+2 \alpha \delta$, then one has $1-\cos \kappa s \leq \delta$, and hence that $s \in J$. Thus

$$
\frac{1}{2 T} \int_{0}^{T} \frac{d s}{A(s)} \leq \frac{1}{2 T} \int_{[0, T] \backslash J} \frac{d s}{1+2 \alpha \delta}+\frac{1}{2 T} \int_{[0, T] \cap J} d s
$$

and it follows from (4.39) and from the structure (4.38) of $J$ that

$$
\gamma=\lim _{T \rightarrow \infty} \frac{1}{2 T} \int_{0}^{T} \frac{d s}{A(s)} \leq \frac{1}{2(1+2 \alpha \delta)}+\frac{\varepsilon}{2 \pi} .
$$


Regarding $\gamma$ as a function of $\alpha$, we now have from (4.40) that

$$
\limsup _{\alpha \rightarrow \infty} \gamma \leq \frac{\varepsilon}{2 \pi}
$$

and hence that $\gamma \rightarrow 0$ as $\alpha \rightarrow \infty$, as $\varepsilon$ can be taken arbitrarily small.

In addition to the analytical dependence of $\Gamma$ on $c \neq 0$, this function also depends at least continuously on the direction parameter $\theta \in \mathbb{R}$, again for $c \neq 0$. On the other hand, the dependence of the limits $\Gamma(0 \pm)= \pm \gamma$ on $\theta$ is rather subtle. In particular, the quantity $\gamma=\gamma(\theta)$ in (4.29), considered as a function of $\theta$, is continuous (in fact, constant) at those $\theta \in \mathbb{R}$ for which $\tan \theta$ is irrational, and is discontinuous at those $\theta \in \mathbb{R}$ for which $\tan \theta$ is rational or infinite. To see this, first let

$$
\begin{aligned}
B\left(\theta_{1}, \theta_{2}\right) & =\left(1+4 \alpha-\alpha\left(e^{i \theta_{1}}+e^{-i \theta_{1}}+e^{i \theta_{2}}+e^{-i \theta_{2}}\right)\right)^{-1} \\
& =(1+4 \alpha)^{-1} \sum_{k=0}^{\infty}(\alpha /(1+4 \alpha))^{k}\left(e^{i \theta_{1}}+e^{-i \theta_{1}}+e^{i \theta_{2}}+e^{-i \theta_{2}}\right)^{k} \\
& =\sum_{m=-\infty}^{\infty} \sum_{n=-\infty}^{\infty} \beta_{m, n} e^{i\left(m \theta_{1}+n \theta_{2}\right)},
\end{aligned}
$$

where we note the above sums are all absolutely convergent, and we also see that $\beta_{m, n}>0$ for all $m, n \in \mathbb{Z}$. From the definition (4.18) of $A(s)$, we have that $1 / A(s)=$ $B(\kappa s, \sigma s)$, so we may substitute (4.41) into (4.29), and express $\gamma=\gamma(\theta)$ in terms of the Fourier coefficients $\beta_{m, n}$, obtaining

$$
\begin{aligned}
\gamma=\gamma(\theta) & =\lim _{T \rightarrow \infty} \frac{1}{2 T} \int_{0}^{T} \sum_{m=-\infty}^{\infty} \sum_{n=-\infty}^{\infty} \beta_{m, n} e^{i(m \kappa+n \sigma) s} d s \\
& =\frac{1}{2} \sum_{m \kappa+n \sigma=0} \beta_{m, n} .
\end{aligned}
$$

First suppose that $\tan \theta=\sigma / \kappa$ is irrational. Then the final sum in (4.42) has only a single term,

$$
\gamma(\theta)=\frac{\beta_{0,0}}{2}
$$

On the other hand, suppose that $\tan \theta$ is either rational or infinite. In this case there exists a quantity $\nu>0$, and integers $M, N$, such that

$$
\kappa=M \nu, \quad \sigma=N \nu
$$

and such that $M$ and $N$ have no common factor, that is, their greatest common divisor is $\operatorname{gcd}(M, N)=1$. In fact, this $\nu$ is the same quantity as in (2.2). Then $m \kappa+n \sigma=(m M+n N) \nu$ vanishes if and only if $m=-N k$ and $n=M k$ for some integer $k$. We therefore have that

$$
\gamma(\theta)=\frac{1}{2} \sum_{k=-\infty}^{\infty} \beta_{-N k, M k} .
$$


Note that the quantity (4.45), for $\tan \theta$ rational, is strictly greater than the corresponding quantity (4.43) for $\tan \theta$ irrational.

The quantity $\gamma(\theta)$ is given as a sum (4.43) or (4.45) of the coefficients $\beta_{m, n}$, and in turn each $\beta_{m, n}$ is given as an infinite series obtained by a binomial expansion of the middle line in (4.41). Interestingly, the quantity $\gamma(\theta)$ can also be evaluated directly, in some cases explicitly, without resorting to an infinite series. To see this, let

$$
\begin{aligned}
\widetilde{B}(w, z) & =\left(1+4 \alpha-\alpha\left(w+w^{-1}+z+z^{-1}\right)\right)^{-1} \\
& =\sum_{m=-\infty}^{\infty} \sum_{n=-\infty}^{\infty} \beta_{m, n} w^{m} z^{n}
\end{aligned}
$$

so that $B\left(\theta_{1}, \theta_{2}\right)=\widetilde{B}\left(e^{i \theta_{1}}, e^{i \theta_{2}}\right)$, where the sum in (4.46) is absolutely convergent, at least for complex $w$ and $z$ in a neighborhood of the unit circle $S^{1} \subseteq \mathbb{C}$. Assuming that $\tan \theta$ is either rational or infinite, and with $M$ and $N$ as above, we have directly from (4.46) that

$$
\begin{aligned}
\frac{1}{4 \pi i} \int_{S^{1}} z^{-1} \widetilde{B}\left(z^{M}, z^{N}\right) d z & =\frac{1}{4 \pi i} \int_{S^{1}} \sum_{m=-\infty}^{\infty} \sum_{n=-\infty}^{\infty} \beta_{m, n} z^{M m+N n-1} d z \\
& =\frac{1}{2} \sum_{M m+N n=0} \beta_{m, n}=\frac{1}{2} \sum_{k=-\infty}^{\infty} \beta_{-N k, M k}=\gamma(\theta) .
\end{aligned}
$$

The first integral in (4.47) is a contour integral of a rational function, which can in principle be evaluated with the residue theorem. We denote the integrand there by $Q(z)$, that is,

$$
Q(z)=z^{-1} \widetilde{B}\left(z^{M}, z^{N}\right)=\left((1+4 \alpha) z-\alpha\left(z^{M+1}+z^{-M+1}+z^{N+1}+z^{-N+1}\right)\right)^{-1} .
$$

For the case of $\tan \theta=0$ we have $M=1$ and $N=0$, which yields

$$
Q(z)=-\alpha^{-1}\left(z^{2}-\left(\alpha^{-1}+2\right) z+1\right)^{-1} .
$$

One easily checks that $Q$ has two poles $z=z_{ \pm}=\left(\alpha^{-1}+2 \pm\left(\alpha^{-2}+4 \alpha^{-1}\right)^{1 / 2}\right) / 2$, and that $0<z_{-}<1<z_{+}$. Therefore $Q(z)=-\alpha^{-1}\left(z-z_{-}\right)^{-1}\left(z-z_{+}\right)^{-1}$, and from the first integral in (4.47)

$$
\gamma(0)=\frac{1}{2 \alpha\left(z_{+}-z_{-}\right)}=\frac{1}{2(1+4 \alpha)^{1 / 2}} .
$$

A similar calculation of $\gamma(\theta)$ can be made for $\theta=\pi / 4$, that is, with $\tan \theta=1$. Alternatively, the change of variables $\psi(\xi)=\varphi\left(2^{-1 / 2} \xi\right)$ takes equation (1.16) with $\theta=\pi / 4$ into equation (1.17), as described earlier, and this implies that $\gamma(\pi / 4)$ equals the value of $\gamma(0)$ with $2 \alpha$ in place of $\alpha$. That is,

$$
\gamma(\pi / 4)=\frac{1}{2(1+8 \alpha)^{1 / 2}}
$$


Calculation of $\gamma(\theta)$ for values of $\theta$ with $\tan \theta$ rational but with $\theta$ not an integer multiple of $\pi / 4$ leads generally to a rational function $Q$ with a denominator which is a polynomial of degree greater than two. The calculation of the poles and residues of $Q$, and hence of the quantity $\gamma(\theta)$, rapidly becomes unwieldy.

If $\tan \theta$ is irrational, then (4.43) holds, so we wish only to calculate $\beta_{0,0}$. To this end, we have from (4.46) for any fixed $w \in S^{1}$ that

$$
\begin{aligned}
\frac{1}{2} \sum_{m=-\infty}^{\infty} \beta_{m, 0} w^{m} & =\frac{1}{4 \pi i} \int_{S^{1}} z^{-1} \widetilde{B}(w, z) d z \\
& =\frac{-\alpha^{-1}}{4 \pi i} \int_{S^{1}}\left(z^{2}-\left(\alpha^{-1}+4-w-w^{-1}\right) z+1\right)^{-1} d z \\
& =\frac{1}{2 \alpha\left(z_{+}-z_{-}\right)},
\end{aligned}
$$

by a calculation similar to one above, where here

$$
z_{ \pm}=z_{ \pm}(w)=\frac{1}{2}\left(\alpha^{-1}+4-w-w^{-1} \pm\left(\left(\alpha^{-1}+4-w-w^{-1}\right)^{2}-4\right)^{1 / 2}\right)
$$

are the poles of the integrand in (4.48), and where $0<\left|z_{-}(w)\right|<1<\left|z_{+}(w)\right|$. We have therefore from (4.43) and (4.48) that

$$
\begin{aligned}
\gamma(\theta)=\frac{\beta_{0,0}}{2} & =\frac{1}{4 \pi i} \int_{S^{1}} \frac{d w}{\alpha w\left(z_{+}(w)-z_{-}(w)\right)} \\
& =\frac{1}{4 \pi i} \int_{S^{1}} \frac{d w}{\left(\left(w+4 \alpha w-\alpha w^{2}-\alpha\right)^{2}-4 \alpha^{2} w^{2}\right)^{1 / 2}}
\end{aligned}
$$

One observes that the final integral in (4.49), with further simplification, can be expressed in terms of complete elliptic integrals, to yield an expression for $\gamma(\theta)$ in closed form. However, we refrain from further calculations in this direction.

As will become clearer with our further analysis, the lattice system (1.1) with nonlinearity (3.1) can support a traveling wave (1.15), (1.11) (at least one which is monotone in the wave parameter $\xi$ ) with speed $c \neq 0$, in the direction $e^{i \theta}$, only when the detuning parameter $a$ satisfies $|a-1 / 2|>\gamma=\gamma(\theta)$. That is, $a$ must be sufficiently far away from the value $1 / 2$ at which the nonlinearity is symmetric. For $|a-1 / 2| \leq \gamma(\theta)$, one expects instead a standing wave, that is, wave speed $c=0$. The range $[1 / 2-\gamma(\theta), 1 / 2+\gamma(\theta)]$ of parameter values at which no nontrivial traveling wave occurs is the range of so-called propagation failure. Our results therefore will show that for the ideal nonlinearity (3.1), propagation failure occurs for every direction $e^{i \theta}$, although the effect is more pronounced for angles of rational slope, as in this case $\gamma(\theta)$ is larger than for angles of irrational slope.

Remark. Table 1 presents the values of $\gamma(\theta)$ for several choices of $\theta$, namely, when $\tan \theta=0,1$, and 0.1 , and also for $\tan \theta$ irrational. A range of values of $\alpha$ is chosen. Note in particular how $\gamma(\theta)$ for $\tan =0.1$ is very close to the corresponding value for $\tan \theta$ irrational.

Remark. Although the limit $\Gamma(0+)=\gamma(\theta)$ is independent of $\theta$, so long as $\tan \theta$ is irrational, this is not generally the case for $\Gamma(c)$ for $c \neq 0$. Denoting $\Gamma(c)=\Gamma(c, \theta)$, and taking two values $\theta_{1}$ and $\theta_{2}$ where both $\tan \theta_{1}$ and $\tan \theta_{2}$ are irrational, one 
TABLE 1

Values of $\gamma(\theta)$ for various values of $\theta$ and $\alpha$.

\begin{tabular}{|c|c|c|c|c|c|}
\hline \multicolumn{6}{|c|}{$\tan \theta$} \\
\hline & & 0.0 & 1.0 & 0.1 & Irrational \\
\hline \multirow{10}{*}{$\alpha$} & 0.1 & 0.42257713 & 0.37267800 & 0.36478641 & 0.36478641 \\
\hline & 0.2 & 0.37267800 & 0.31008684 & 0.29326612 & 0.29326612 \\
\hline & 0.3 & 0.33709993 & 0.27116307 & 0.24776696 & 0.24776695 \\
\hline & 0.4 & 0.31008684 & 0.24397502 & 0.21584215 & 0.21584210 \\
\hline & 0.5 & 0.28867513 & 0.22360680 & 0.19201185 & 0.19201168 \\
\hline & 0.6 & 0.27116307 & 0.20761370 & 0.17344295 & 0.17344253 \\
\hline & 0.7 & 0.25649459 & 0.19462474 & 0.15850807 & 0.15850723 \\
\hline & 0.8 & 0.24397502 & 0.18380366 & 0.14619956 & 0.14619805 \\
\hline & 0.9 & 0.23312620 & 0.17460757 & 0.13585715 & 0.13585470 \\
\hline & 1.0 & 0.22360680 & 0.16666667 & 0.12702862 & 0.12702492 \\
\hline
\end{tabular}

generally expects that $\Gamma\left(c, \theta_{1}\right) \neq \Gamma\left(c, \theta_{2}\right)$ for $c \neq 0$, despite the fact that these two quantities approach the same limit as $c \rightarrow 0$.

Remark. We believe that the pathological behavior of $\gamma(\theta)$ described above, namely, that $\gamma(\theta)$ is continuous where $\tan \theta$ is irrational and discontinuous where $\tan \theta$ is rational or infinite, is not an artifact of the discontinuity of $f$. That is, we believe the same phenomenon occurs for smooth nonlinearities $f$ such as the cubic function (1.3). We expect that a rigorous mathematical treatment of this problem would entail a delicate, and probably difficult, analysis of the functional differential equation (1.16) in the singularly perturbed case $c \rightarrow 0$.

We now return to our task of justifying the calculation of the function $\varphi$. In particular, we must show that $\varphi$ satisfies (4.24) whenever (4.25) holds with $c \neq 0$, in order to conclude that $\varphi$ indeed satisfies the original equation (1.16) of interest. We shall in fact show that $\varphi$ is a strictly increasing function, thereby establishing (4.24).

LEMma 4.4. The function $R$, in (4.13), possesses a simple root $s=-i z_{0}$ with $z_{0}>0$, and we have $i R^{\prime}\left(-i z_{0}\right)>0$. Moreover, there exists $\varepsilon_{0}>0$ such that if $s \in \mathbb{C}$ is any other root of $R$ satisfying $\operatorname{Im} s \leq 0$, then in fact $\operatorname{Im} s<-z_{0}-\varepsilon_{0}$.

Proof. Consider

$$
R(-i z)=-c z+1+2 \alpha(2-\cosh \kappa z-\cosh \sigma z) .
$$

The expression (4.50), considered as a function of $z \geq 0$, is positive at $z=0$, negative for large $z$, and has a negative second derivative with respect to $z$, for all $z \geq 0$. This implies the existence of a unique $z_{0}>0$ at which $R\left(-i z_{0}\right)=0$, and moreover implies that $s=-i z_{0}$ is a simple root of $R$, and that the quantity $i R^{\prime}\left(-i z_{0}\right)$ is positive. Let us also note that $R(-i z)>0$ whenever $0 \leq z<z_{0}$.

Consider now any other $s=u-i z$, where $u$ is real, $z \geq 0$, and $R(s)=0$. From (4.13) we have that

$$
\begin{aligned}
0=R(u-i z)= & -i c u-c z+1+2 \alpha(2-\cos \kappa u \cosh \kappa z-\cos \sigma u \cosh \sigma z) \\
& -2 i \alpha(\sin \kappa u \sinh \kappa z+\sin \sigma u \sinh \sigma z)
\end{aligned}
$$

after a calculation, and taking the real part of (4.51) gives

$$
0=-c z+1+2 \alpha(2-\cos \kappa u \cosh \kappa z-\cos \sigma u \cosh \sigma z) \geq R(-i z),
$$


where we note the formula (4.50). Necessarily $z \geq z_{0}$, as $R(-i z)>0$ if $0 \leq z<z_{0}$ holds.

All that remains now is to show that $z>z_{0}+\varepsilon_{0}$ for some $\varepsilon_{0}$ independent of the root $s$, unless $s=-i z_{0}$. Taking the imaginary part

$$
0=-c u-2 \alpha(\sin \kappa u \sinh \kappa z+\sin \sigma u \sinh \sigma z)
$$

of (4.51) first shows that there is a bound $|u| \leq K$ on the real part of all roots $s$ in the strip $z_{0} \leq z \leq z_{0}+1$, and so this strip contains only finitely many roots of $R$. It is therefore enough to prove that the only root of $R$ on the horizontal line $\operatorname{Im} s=-z_{0}$ is the root $s=-z_{0}$ obtained above. Suppose, therefore, that $z=z_{0}$. Then the inequality in (4.52) is an equality, and so $\cos \kappa u=\cos \sigma u=1$, by (4.50). Therefore $\sin \kappa u=\sin \sigma u=0$, and so $u=0$ by (4.53). Thus we have $s=-i z_{0}$, as desired.

COROLlary 4.5. The solution $\varphi$ of (4.3), given by (4.20), satisfies

$$
\varphi(\xi)=C e^{z_{0} \xi}+O\left(e^{\left(z_{0}+\varepsilon_{0}\right) \xi}\right) \text { as } \xi \rightarrow-\infty,
$$

where $z_{0}$ and $\varepsilon_{0}$ are as in Lemma 4.4 and where $C>0$. The derivative $\varphi^{\prime}$ satisfies the formula

$$
\varphi^{\prime}(\xi)=C z_{0} e^{z_{0} \xi}+O\left(e^{\left(z_{0}+\varepsilon_{0}\right) \xi}\right) \text { as } \xi \rightarrow-\infty
$$

obtained by formally differentiating (4.54). The analogous results for $\xi \rightarrow \infty$, obtained from (4.21), hold. In particular, there exists $T>0$ such that

$$
\varphi^{\prime}(\xi)>0 \text { for }|\xi| \geq T \text {. }
$$

Proof. We use the contour integral formula (4.15), which is valid for all sufficiently small $\varepsilon>0$. Arguing much as before, we shift the contour $\operatorname{Im} s=-\varepsilon$ downward, past the pole at $s=-i z_{0}$, to the contour $\operatorname{Im} s=-z_{0}-\varepsilon_{0}$, where here $z_{0}$ and $\varepsilon_{0}$ are as in Lemma 4.4. We obtain

$$
\varphi(\xi)=-\left.\frac{e^{i s \xi}}{s R^{\prime}(s)}\right|_{s=-i z_{0}}+\frac{1}{2 \pi i} \int_{-i\left(z_{0}+\varepsilon_{0}\right)-\infty}^{-i\left(z_{0}+\varepsilon_{0}\right)+\infty} \frac{e^{i s \xi}}{s R(s)} d s=C e^{z_{0} \xi}+O\left(e^{\left(z_{0}+\varepsilon_{0}\right) \xi}\right),
$$

where $C=1 /\left(i z_{0} R^{\prime}\left(-i z_{0}\right)\right)$ is positive, by Lemma 4.4. This proves (4.54).

To prove the asymptotic formula (4.55) for the derivative, one first notes from the differential equation (4.3) that

$$
\varphi^{\prime}(\xi)=K e^{z_{0} \xi}+O\left(e^{\left(z_{0}+\varepsilon_{0}\right) \xi}\right) \text { as } \xi \rightarrow-\infty,
$$

for some real $K$. Integrating (4.57) from $-\infty$ to $\xi$ must yield (4.54), and so necessarily $K / z_{0}=C$.

Finally, as in the statement of the result, the symmetry relation (4.21) easily yields the analogues of (4.54), (4.55), as $\xi \rightarrow \infty$, and in particular we have (4.56). 口

THEOREM 4.6. The solution $\varphi$ of (4.3), given by (4.20), is strictly increasing on the real line. In particular, $\varphi$ also satisfies (1.16), with (1.11), where the nonlinearity $f$ is given by (3.1).

Proof. We first prove, basically using a maximum principle, that

$$
0<\varphi(\xi)<1
$$


for all $\xi \in \mathbb{R}$. Suppose that (4.58) is false, say $\varphi(\xi) \geq 1$ for some $\xi$. Now certainly (4.58) holds for $|\xi| \geq T$, with $T$ as in (4.56) in Corollary 4.5. Therefore, if we let $\eta=\sup \{\varphi(\xi) \mid \xi \in \mathbb{R}\}$, we have that $\eta \geq 1$, and so there exists $\xi_{0} \in \mathbb{R}$ such that $\varphi\left(\xi_{0}\right)=\eta$ and $\varphi(\xi)<\eta$ for all $\xi<\xi_{0}$ (we take the leftmost point at which $\varphi(\xi)$ achieves its maximum $\eta$ ). Necessarily, $\alpha L \varphi\left(\xi_{0}\right)<0$, by the formula for $L$. Therefore, both the left- and right-hand limits, as $\xi \rightarrow \xi_{0}$, of the right-hand side of the differential equation (4.3) are negative. But then, the two limits $\varphi^{\prime}\left(\xi_{0}-\right)$ and $\varphi^{\prime}\left(\xi_{0}+\right)$ are both nonzero, and have the same sign, and this contradicts the fact that the piecewise $C^{1}$ solution $\eta$ achieves its maximum at $\xi_{0}$. This establishes the inequalities (4.58).

Now, by noting the boundary conditions (1.11) and by using (4.58), we may assume, by increasing $T$ if necessary, that both

$$
\varphi(\xi)<\varphi(T) \text { for } \xi<T, \quad \varphi(\xi)>\varphi(-T) \text { for } \xi>-T
$$

hold. For any $\tau>0$, define the function

$$
\psi_{\tau}(\xi)=\varphi(\xi+\tau)-\varphi(\xi)
$$

and observe, using (4.56), that

$$
\psi_{\tau}(\xi)>0 \text { if either } \xi \geq T \text { or } \xi \leq-T-\tau .
$$

Let us also note that if $\tau>2 T$, then $\psi_{\tau}(\xi)>0$ for all $\xi \in \mathbb{R}$. Indeed, for such $\tau$ this conclusion follows easily from (4.56) and (4.59), using the fact that for each $\xi$ we have that either $\xi<-T$, or else that $\xi+\tau>T$.

Now define the quantity

$$
\tau_{0}=\inf \left\{\tau>0 \mid \psi_{\tau}(\xi)>0 \text { for all } \xi \in \mathbb{R}\right\} .
$$

We certainly have that $\tau_{0} \in[0,2 T]$. If in fact $\tau_{0}=0$, then we have immediately that $\varphi$ is everywhere strictly increasing, and we are done. Let us assume, therefore, that $\tau_{0}>0$, and seek a contradiction.

For simplicity write $\psi_{*}(\xi)=\psi_{\tau_{0}}(\xi)$. Certainly $\psi_{*}(\xi) \geq 0$ for all $\xi \in \mathbb{R}$. Also, in light of (4.60), there exists a point $\xi_{0} \in\left[-T-\tau_{0}, T\right]$ such that $\psi_{*}\left(\xi_{0}\right)=0$. Since all such points at which $\psi_{*}$ vanishes lie in that interval, we may without loss assume that $\xi_{0}$ is chosen as the leftmost such point, that is, we have that $\psi_{*}(\xi)>0$ for $\xi<\xi_{0}$, and hence that $\alpha L \psi_{*}\left(\xi_{0}\right)>0$. Finally, we note that since $\varphi$ satisfies the differential equation (4.3), the function $\psi_{*}$ satisfies the equation

$$
-c \psi_{*}^{\prime}(\xi)=\alpha L \psi_{*}(\xi)-\psi_{*}(\xi)+h\left(\xi+\tau_{0}\right)-h(\xi)
$$

on the line.

We now argue much as in the early part of the proof. In particular, we see that both the left- and right-hand limits of the right-hand side of equation (4.61) are positive, at $\xi=\xi_{0}$. This contradicts the fact that $\psi_{*}$ achieves its minimum at $\xi_{0}$, and thereby proves the theorem.

Remark. With minor modifications, the above proof shows that if a solution $\varphi$ of (1.16), (1.11), with $c \neq 0$, and continuous $f: \mathbb{R} \rightarrow \mathbb{R}$ satisfying $f(u) \geq 0$ for $u \geq 1$ and $f(u) \leq 0$ for $u \leq 1$, is strictly increasing for $|\xi| \geq T$ for some $T>0$, then it is increasing for all $\xi \in \mathbb{R}$. (Note that this class of $f$ includes the cubic (1.3).) In practice, one might expect to establish that a given solution was strictly increasing for large $|\xi|$, by considering the linearization of (1.16) around limiting values $\varphi( \pm \infty)$. This 
approach, for a class of equations with retarded arguments (time-delay systems), has been taken in [7], and more recently in [22], [23], for mixed equations such as (1.16), although a considerable amount of analysis is required for this.

We close this section by studying the function $\Gamma$, which provides the relation (4.26) between the wave speed $c$ and the detuning parameter $a$. In particular, we prove a strictly monotone relation between these parameters. This implies that $c \neq 0$ is uniquely determined from $a$ in the range $\gamma<|a-1 / 2|<1 / 2$ and thereby addresses some of our earlier concerns about the uniqueness of traveling waves.

THEOREM 4.7. The function $\Gamma$ given by (4.26) satisfies

$$
\Gamma^{\prime}(c)>0 \text { for all real } c \neq 0 \text {. }
$$

In particular, for each $a \in(0,1),(4.25)$ has exactly one solution $c \neq 0$ if and only if $|a-1 / 2|>\gamma$ and has no solution $c \neq 0$ if and only if $|a-1 / 2| \leq \gamma$. The solution $c=c(a)$ depends analytically on $a$, for $|a-1 / 2|>\gamma$.

Proof. While one may differentiate the expression involving the integral in (4.26) directly, the resulting formula for $\Gamma^{\prime}(c)$ unfortunately provides no insight as to the sign of this quantity. We therefore take an alternate approach, and work directly with the differential equation, and calculate $D_{c} \varphi(0, c)$. Our techniques are related to, and motivated by, those connected with the so-called Mel'nikov method [29] (see also [6]) for studying homo- and heteroclinic orbits of dynamical systems. In particular, this general approach has been used for equations with retarded arguments in [7] and [17], and very recently [23] in this spirit for mixed equations of a general form.

Let $c \neq 0$ be fixed. We have that $\Gamma(c)=\varphi(0, c)-1 / 2$, where $\varphi(\xi, c)$ denotes the solution (4.20) of (4.3), (1.11), and so $\Gamma^{\prime}(c)=\psi(0, c)$, where $\psi=D_{c} \varphi$, as in Proposition 4.2. Denote $\eta(\xi)=\varphi^{\prime}(-\xi, c)$. Then the function $\eta$ is $C^{1}$ on the interval $(-\infty, 0]$ and $[0, \infty)$ and undergoes a jump discontinuity

$$
\eta(0+)-\eta(0-)=\varphi^{\prime}(0-, c)-\varphi^{\prime}(0+, c)=\frac{1}{c}
$$

at the origin, by (4.3). As $\eta(\xi)=\varphi^{\prime}(-\xi, c)$ and hence $\eta(-\xi)=\varphi^{\prime}(\xi, c)=\varphi^{\prime}(-\xi,-c)$ by (4.21), we have by Corollary 4.5 that the function $\eta$ decays exponentially at $\pm \infty$. Differentiating (4.3) gives

$$
c \eta^{\prime}(\xi)=\alpha L \eta(\xi)-\eta(\xi)
$$

for $\xi \neq 0$, and it follows directly from (4.64) that $\eta^{\prime}$ also decays exponentially at $\pm \infty$. In particular, both $\eta$ and $\eta^{\prime}$ are integrable functions.

Now multiply both sides of (4.22) by $\eta(\xi)$ and integrate the resulting expression from $-\infty$ to $\infty$. Denoting $\varphi(\xi)=\varphi(\xi, c)$ and $\psi(\xi)=\psi(\xi, c)$, we obtain

$$
\begin{aligned}
-\int_{-\infty}^{\infty}\left(\varphi^{\prime}(\xi)+c \psi(\xi)\right) \eta(\xi) d \xi & =\int_{-\infty}^{\infty}(\alpha L \psi(\xi)-\psi(\xi)) \eta(\xi) d \xi \\
& =\int_{-\infty}^{\infty}(\alpha L \eta(\xi)-\eta(\xi)) \psi(\xi) d \xi
\end{aligned}
$$

by a small calculation. Also, integrating by parts on the two intervals $(-\infty, 0]$ and $[0, \infty)$ and using (4.63) and (4.64) give

$$
-c \int_{-\infty}^{\infty} \psi^{\prime}(\xi) \eta(\xi) d \xi=-c \psi(0)(\eta(0-)-\eta(0+))+c\left(\int_{-\infty}^{0}+\int_{0}^{\infty}\right) \psi(\xi) \eta^{\prime}(\xi) d \xi
$$

$$
=\psi(0)+\int_{-\infty}^{\infty} \psi(\xi)(\alpha L \eta(\xi)-\eta(\xi)) d \xi .
$$


Combining (4.65) and (4.66) and simplifying, we obtain

$$
\Gamma^{\prime}(c)=\psi(0)=\int_{-\infty}^{\infty} \varphi^{\prime}(\xi) \eta(\xi) d \xi=\int_{-\infty}^{\infty} \varphi^{\prime}(\xi) \varphi^{\prime}(-\xi) d \xi
$$

The right-hand integral of (4.67) is nonnegative by Theorem 4.6. In fact, for large $|\xi|$ the integrand $\varphi^{\prime}(\xi) \varphi^{\prime}(-\xi)$ is strictly positive, by (4.56) of Corollary 4.5. Thus $\Gamma^{\prime}(c)>0$, as claimed.

The remaining claims of the theorem now follow directly from (4.62) and from Theorem 4.3, using in particular the implicit function theorem.

Our construction of discrete traveling wave solutions of equation (1.1), with $f$ as in (3.1), is easily extended to a broader class of nonlinearities of the same general type. Specifically, consider

$$
f(u)=u-\sum_{k=1}^{n} b_{k} h\left(u-a_{k}\right)
$$

where $0<a_{1}<a_{2}<\cdots<a_{n}<1$ and where

$$
\sum_{k=1}^{n} b_{k}=1, \quad b_{k}>0 \text { for each } k=1,2, \ldots, n .
$$

When $n=1$, of course, the function $f$ in (4.68) reduces to the one (3.1) considered above. For any quantities $\xi_{1}<\xi_{2}<\cdots<\xi_{n}$ and $c \neq 0$, denote

$$
\psi(\xi, \widehat{\xi}, \widehat{b}, c)=\sum_{k=1}^{n} b_{k} \varphi\left(\xi-\xi_{k}, c\right),
$$

where $\varphi(\xi, c)$ is the solution (4.20) of (4.3) and where we denote $\widehat{\xi}=\left(\xi_{1}, \xi_{2}, \ldots, \xi_{n}\right)$ and $\widehat{b}=\left(b_{1}, b_{2}, \ldots, b_{n}\right)$. The function $\psi$ is increasing in $\xi$, since all the coefficients $b_{k}$ are positive, and one easily sees that $\psi$ satisfies (1.16) with (1.11), with $f$ as in (4.68), if and only if $\psi\left(\xi_{j}, \widehat{\xi}, \widehat{b}, c\right)=a_{j}$ for each $j$; that is,

$$
\sum_{k=1}^{n} b_{k} \varphi\left(\xi_{j}-\xi_{k}, c\right)=a_{j}, \quad j=1,2, \ldots, n
$$

holds. Without loss we may normalize $\xi_{n}=0$ by a translation of $\xi$. With this, and with the quantities $a_{k}$ and $b_{k}$ in the nonlinearity $f$ given, observe that equation (4.69) is a system of $n$ equations in the $n$ unknowns $\xi_{1}, \xi_{2}, \ldots, \xi_{n-1}$, and $c$, so one expects generically at most a finite number of solutions. In contrast to the case $n=1$ considered earlier, however, it is not ruled out that for some values $a_{k}$ there might be multiple solutions $(\widehat{\xi}, c)$ to (4.69). See the related results in [2] and [38] for the spatially continuous (nonlattice) case.

Quite generally, let $\Phi(\widehat{\xi}, c)$ denote the expression in the left-hand side of (4.69), considered as an element of $\mathbb{R}^{n}$. One wishes to understand the mapping $(\widehat{\xi}, c) \rightarrow$ $\Phi(\widehat{\xi}, c)$, and in particular the range of $\Phi$ in $\mathbb{R}^{n}$ and the number of times a given value $a \in \mathbb{R}^{n}$ is assumed by $\Phi$. Theorem 4.7 , in particular, has no clear generalization to the case $n>1$, and one would expect appropriate counterexamples to exist.

One is also interested in the dependence of $\Phi$ on the direction $e^{i \theta}$ of the traveling wave, and of course one expects behavior at least as complicated as in the case $n=1$. 
5. Zero wave speed. In this section we consider solutions of (1.16), (1.11) with wave speed $c=0$. An essential difference from the case of nonzero $c$ is that, in view of (1.15), one is only concerned with values $\varphi(\xi)$ for $\xi \in D$, where $D \subseteq \mathbb{R}$ is the countable set (2.1). Recall that if the ratio $\tan \theta=\sigma / \kappa$ is irrational, then $D$ is a dense subset of $\mathbb{R}$, while if $\tan \theta$ is rational or infinite, then (2.2) holds for some quantity $\nu>0$, and $D$ is a discrete subset of $\mathbb{R}$.

In the spirit of the previous section, we shall construct solutions of equation (1.16) which satisfy

$$
\varphi(\xi)<a \text { for } \xi \in\left(-\infty, \xi_{0}\right) \cap D, \quad \varphi(\xi)>a \text { for } \xi \in\left(\xi_{0}, \infty\right) \cap D,
$$

which is a condition analogous to (4.1). In view of the special role of the set $D$, we do not necessarily take $\xi_{0}=0$ as the point where $\varphi(\xi)$ crosses the value $a$ but rather consider arbitrary $\xi_{0} \in \mathbb{R}$. In particular, we may have either $\xi_{0} \in D$ or $\xi_{0} \notin D$. In fact, when $\tan \theta$ is irrational we shall obtain uncountably many solutions to (1.16), (1.11), with $c=0$, parameterized, basically, by the equivalence classes of $\xi_{0}$ modulo the set $D$. Of course, a solution $\varphi$ of (1.16) on $D$ that satisfies (5.1) also satisfies the inhomogeneous linear equation

$$
0=\alpha L \varphi(\xi)-\varphi(\xi)+h\left(\xi-\xi_{0}\right),
$$

since $f(\varphi(\xi)) \subseteq \varphi(\xi)-h\left(\xi-\xi_{0}\right)$ for $\xi \in D$.

Although we need only consider solutions of (1.16) which are defined for $\xi \in D$, that is, solutions $\varphi: D \rightarrow \mathbb{R}$, we shall in fact first construct solutions $\varphi: \mathbb{R} \rightarrow \mathbb{R}$ of (5.2) defined on the whole real line and then consider their restriction to $D$. We shall show there is no loss of generality in this approach, at least within the class of bounded solutions satisfying (5.1).

We shall obtain our solutions by taking limits $c \rightarrow 0$ of the traveling wave solutions constructed in the previous section. Before doing this, we introduce a bit of notation which will prove useful. Let $\tau \in \mathbb{R}$ (typically, we shall take $\tau \in[0,1]$ ), and define the function $h_{\tau}: \mathbb{R} \rightarrow \mathbb{R}$ by

$$
h_{\tau}(u)= \begin{cases}h(u) & \text { for } \quad u \neq 0, \\ \tau & \text { for } \quad u=0 .\end{cases}
$$

That is, $h_{\tau}$ is just the Heaviside function (3.2) with the specific value $\tau$ assigned to $h_{\tau}(0)$. Unlike the set-valued function $h$, each function $h_{\tau}$ is single-valued. Also set

$$
h_{-}=h_{0}, \quad h_{+}=h_{1},
$$

the function $h_{\tau}$ with $\tau=0$ and 1 , and observe that $h_{ \pm}(u)=h(u \pm)$ are the left- and right-hand limits of $h$.

Now let $\varphi(\xi, c)$ denote the solution to (4.3), (1.11) for $c \neq 0$, and consider a sequence $c_{n} \rightarrow 0$, with each $c_{n} \neq 0$. Because, by Theorem 4.6, the solution $\varphi(\xi, c)$ is increasing and uniformly bounded in $\xi$, after possibly passing to a subsequence we have the limit

$$
\varphi\left(\xi, c_{n}\right) \rightarrow \varphi_{*}(\xi)
$$

pointwise, for all but countably many $\xi \in \mathbb{R}$, for some nondecreasing limit function $\varphi_{*}: \mathbb{R} \rightarrow[0,1]$. The function $\varphi_{*}$ need not be continuous, and in fact we shall shortly 
show that it necessarily has discontinuities. As $\varphi_{*}$ is monotone, all of its discontinuities are jumps, and so we may define two other functions $\varphi_{ \pm}: \mathbb{R} \rightarrow[0,1]$ by

$$
\varphi_{-}(\xi)=\varphi_{*}(\xi-), \quad \varphi_{+}(\xi)=\varphi_{*}(\xi+)
$$

the left- and right-hand limits, respectively, of $\varphi_{*}$ at each point $\xi \in \mathbb{R}$. The functions $\varphi_{-}$and $\varphi_{+}$are left- and right-continuous, respectively, on $\mathbb{R}$, and they equal $\varphi_{*}$ almost everywhere. Although it is not apparent here, the functions $\varphi_{ \pm}$are independent of the sequence $c_{n}$ used in their definition. We prove this fact below.

In order to take the limit $c_{n} \rightarrow 0$ in the differential equation (4.3), we must first write that equation in integrated form. Fix any $\xi \in \mathbb{R}$ and integrate (4.3) to obtain

$$
-c(\varphi(\xi)-\varphi(0))=\int_{0}^{\xi} \alpha L \varphi(\zeta)-\varphi(\zeta)+h(\zeta) d \zeta .
$$

Upon taking the limit $c=c_{n} \rightarrow 0$ in (5.6), with $\varphi(\zeta)=\varphi\left(\zeta, c_{n}\right)$, we obtain, using the Lebesgue dominated convergence theorem, that

$$
0=\int_{0}^{\xi} \alpha L \varphi_{*}(\zeta)-\varphi_{*}(\zeta)+h(\zeta) d \zeta
$$

for all $\xi$. Differentiating (5.7) now yields the equation

$$
0=\alpha L \varphi_{*}(\xi)-\varphi_{*}(\xi)+h(\xi),
$$

which holds for almost every $\xi \in \mathbb{R}$.

Equation (5.8), involving the function $\varphi_{*}$, is inadequate for our purposes since it only holds almost everywhere in $\xi$. In particular, one is concerned that equation (5.8) may fail at all points of the set $D$, which has measure zero. To address this situation, consider the functions $\varphi_{ \pm}$. We certainly have that

$$
0=\alpha L \varphi_{ \pm}(\xi)-\varphi_{ \pm}(\xi)+h_{ \pm}(\xi)
$$

holds almost everywhere, with $h_{ \pm}$as in (5.3), (5.4), since $L \varphi_{ \pm}, \varphi_{ \pm}$, and $h_{ \pm}$agree almost everywhere with $L \varphi_{*}, \varphi_{*}$, and $h$, respectively. In fact, (5.9), with either choice + or - of \pm , holds for all $\xi \in \mathbb{R}$. One sees this directly from the fact that both $\varphi_{-}$ and $h_{-}$are left-continuous on $\mathbb{R}$ and that both $\varphi_{+}$and $h_{+}$are right-continuous on $\mathbb{R}$, so (5.9) may be obtained at every $\xi \in \mathbb{R}$ by taking the appropriate one-sided limit.

Let us define the linear combination

$$
\varphi_{\tau}(\xi)=\tau \varphi_{+}(\xi)+(1-\tau) \varphi_{-}(\xi) .
$$

One now has from (5.9) and the definition (5.3) of $h_{\tau}$ that the equation

$$
0=\alpha L \varphi_{\tau}(\xi)-\varphi_{\tau}(\xi)+h_{\tau}(\xi),
$$

holds for all $\xi \in \mathbb{R}$. We know that the limits $\varphi_{\tau}( \pm \infty)$ exist, by the monotonicity of $\varphi_{\tau}$. We have directly, by taking the limits $\xi \rightarrow \pm \infty$ in (5.10), that

$$
\varphi_{\tau}(-\infty)=0, \quad \varphi_{\tau}(\infty)=1
$$

that is, $\varphi_{\tau}$ satisfies the boundary conditions (1.11). 
Questions of whether the monotonicity of $\varphi_{\tau}$ is strict or not become important. Unlike the case $c \neq 0$ of the previous section, $\varphi_{\tau}$ may be constant on some intervals, as we see in Corollary 5.3, although these cannot be too large, by Theorem 5.1. It is here that the difference between the cases of rational and irrational slope $\tan \theta$ become more pronounced.

THEOREM 5.1. Let $\tau \in[0,1]$. If $\tan \theta$ is irrational, then the function $\varphi_{\tau}$ is strictly increasing on $\mathbb{R}$. If $\tan \theta$ is rational or infinite, then $\varphi_{\tau}(\xi)<\varphi_{\tau}(\xi+\nu)$ for all $\xi \in \mathbb{R}$, where $\nu$ is as in (2.2). In any case, if we define

$$
\varphi_{\tau}\left(\xi, \xi_{0}\right)=\varphi_{\tau}\left(\xi-\xi_{0}\right),
$$

then for any $\tau \in[0,1]$ and $\xi_{0} \in \mathbb{R}$, the function $\varphi_{\tau}\left(\cdot, \xi_{0}\right)$ restricted to the set $D$ is strictly increasing.

Proof. Let $\tau \in[0,1]$ be fixed throughout this proof. We first observe that for such $\tau$, the function $\varphi_{\tau}$ is nondecreasing everywhere on $\mathbb{R}$, as it is a convex combination of the nondecreasing functions $\varphi_{ \pm}$. Now suppose that $I \subseteq \mathbb{R}$ is an interval on which $\varphi_{\tau}$ is constant; that is, $\varphi_{\tau}\left(\xi_{1}\right)=\varphi_{\tau}\left(\xi_{2}\right)$ for all $\xi_{1}, \xi_{2} \in I$. From (5.10) and the formula (2.4) for $L$, we have that

$$
\varphi_{\tau}(\xi+\kappa)=-\varphi_{\tau}(\xi-\kappa)-\varphi_{\tau}(\xi+\sigma)-\varphi_{\tau}(\xi-\sigma)+\frac{1}{\alpha}\left((1+4 \alpha) \varphi_{\tau}(\xi)-h_{\tau}(\xi)\right)
$$

and in particular we see that the right-hand side of (5.13) is nonincreasing for $\xi \in I$ (in particular, since $h_{\tau}$ is nondecreasing, by the choice of $\tau$ ). On the other hand, the left-hand side of (5.13) is nondecreasing for all $\xi \in \mathbb{R}$ and hence is constant for $\xi \in I$. We therefore conclude that $\varphi_{\tau}$ is also constant on the interval $I+\kappa$; that is, $\varphi_{\tau}\left(\xi_{1}\right)=\varphi_{\tau}\left(\xi_{2}\right)$ for all $\xi_{1}, \xi_{2} \in I+\kappa$, where we denote generally $I+\rho=\{\xi+\rho \mid \xi \in I\}$, for any real number $\rho$.

In a similar fashion, we conclude that $\varphi_{\tau}$ is constant on each of the three intervals $I-\kappa$ and $I \pm \sigma$. It now follows directly, using (2.1), that $\varphi_{\tau}$ is constant on the interval $I+\rho$ for each $\rho \in D$. Suppose, in addition, that the interval $I$ on which $\varphi_{\tau}$ is constant contains two distinct points $\xi_{1}, \xi_{2} \in I$, with $\xi_{2}-\xi_{1} \in D$. One then easily sees that the translates $I+\rho$ for $\rho \in D$ cover the real line $\mathbb{R}$, overlapping so that the function $\varphi_{\tau}$ is constant on all of $\mathbb{R}$. This, however, contradicts the limits (5.11), so we conclude that no such points $\xi_{1}, \xi_{2}$ exist.

It follows, therefore, that if $\xi_{1}<\xi_{2}$ are any two real numbers such that $\xi_{2}-\xi_{1} \in D$, then necessarily $\varphi_{\tau}\left(\xi_{1}\right)<\varphi_{\tau}\left(\xi_{2}\right)$ must hold. From this fact, from the density of $D$ if $\tan \theta$ is irrational, and from (2.2) if $\tan \theta$ is rational, the claims of the theorem follow.

Let us observe that for any real $\tau$ and $\xi_{0}$, the function $\varphi(\xi)=\varphi_{\tau}\left(\xi-\xi_{0}\right)$ given by (5.12) satisfies the equation

$$
0=\alpha L \varphi(\xi)-\varphi(\xi)+h_{\tau}\left(\xi-\xi_{0}\right)
$$

for all $\xi \in \mathbb{R}$ and, in particular, for all $\xi \in D$. The next result proves that this is the only such bounded solution of (5.14).

Proposition 5.2. Let $\tau, \xi_{0} \in \mathbb{R}$. Then a bounded function $\varphi: D \rightarrow \mathbb{R}$ satisfies (5.14) for all $\xi \in D$ if and only if $\varphi(\xi)=\varphi_{\tau}\left(\xi, \xi_{0}\right)$ for all $\xi \in D$.

Proof. We have already observed that $\varphi_{\tau}\left(\xi, \xi_{0}\right)$ satisfies $(5.14)$ on $D$, so all that is needed is to prove uniqueness of this solution in the space $l^{\infty}(D)$. Suppose that $\varphi, \widetilde{\varphi} \in l^{\infty}(D)$ are two solutions to (5.14), and let $\psi=\varphi-\widetilde{\varphi}$. Then $\psi$ satisfies the 
homogeneous equation $(\alpha L-I) \psi=0$ in $l^{\infty}(D)$. We wish to show that $\psi$ is the zero element in $l^{\infty}(D)$, and to this end, it suffices to show that $1 \notin \operatorname{spec}(\alpha L)$, where here we regard $L$ as a bounded linear operator on the space $l^{\infty}(D)$.

We may write

$$
L=S_{\kappa}+S_{-\kappa}+S_{\sigma}+S_{-\sigma}-4 I
$$

in terms of the translation operators $S_{\rho}: l^{\infty}(D) \rightarrow l^{\infty}(D)$, defined for each $\rho \in D$ as $S_{\rho} \eta(\xi)=\eta(\xi+\rho)$ for all $\xi \in D$. We observe that $\left\|S_{\rho}\right\|=1$ for the operator norm, and that also $S_{\rho}^{-1}=S_{-\rho}$. From this, and from the spectral mapping theorem, we have that $\operatorname{spec}\left(S_{\rho}\right) \subseteq S^{1}$ and hence that

$$
\operatorname{spec}\left(S_{\rho}+S_{-\rho}-2 I\right) \subseteq[-4,0] .
$$

It follows now from (5.15) and (5.16), and the inclusion (1.4) for commuting operators, that $\operatorname{spec}(\alpha L) \subseteq[-8 \alpha, 0]$. Thus $1 \notin \operatorname{spec}(\alpha L)$, as desired.

Remark. We conclude from the uniqueness claims of Proposition 5.2 that the functions $\varphi_{*}$ (defined almost everywhere) and $\varphi_{ \pm}$and $\varphi_{\tau}$, obtained after taking the limit (5.5) of the traveling waves with nonzero speed are independent of the particular sequence $c_{n} \rightarrow 0$ chosen. In particular, from the symmetry property (4.21), we conclude that $\varphi_{*}(\xi)=1-\varphi_{*}(-\xi)$ almost everywhere and hence that

$$
\varphi_{-}(\xi)=1-\varphi_{+}(-\xi)
$$

for all $\xi \in \mathbb{R}$.

Corollary 5.3. Assume that $\tan \theta$ is either rational or infinite, with $\nu$ as in (2.2). Then for each integer $n$, the function $\varphi_{\tau}$ is constant on the interval $(n \nu,(n+$ 1) $\nu)$.

Proof. Fix $n$, take $\xi_{1}, \xi_{2} \in(n \nu,(n+1) \nu)$, and consider the functions $\varphi_{\tau}\left(\xi,-\xi_{i}\right)=$ $\varphi_{\tau}\left(\xi+\xi_{i}\right)$, for $i=1,2$. We observe that $h_{\tau}\left(\xi+\xi_{1}\right)=h_{\tau}\left(\xi+\xi_{2}\right)$ for all $\xi \in D$, in view of (2.2), and conclude using the uniqueness result of Proposition 5.2 that $\varphi_{\tau}\left(\xi,-\xi_{1}\right)=\varphi_{\tau}\left(\xi,-\xi_{2}\right)$ for all $\xi \in D$. In particular, taking $\xi=0$, we have that $\varphi_{\tau}\left(\xi_{1}\right)=\varphi_{\tau}\left(\xi_{2}\right)$, as desired.

The next result characterizes the locations of the discontinuities of $\varphi_{\tau}$. In particular, when $\tan \theta$ is irrational, we have that $\varphi_{\tau}$ is discontinuous everywhere on the countable dense set $D$.

Proposition 5.4. If $\tau \in[0,1]$, then the left- and right-hand limits $\varphi_{-}(\xi) \leq \varphi_{+}(\xi)$ of $\varphi_{\tau}$ exist at each $\xi \in \mathbb{R}$. The point $\xi$ is a discontinuity of $\varphi_{\tau}$ if and only if $\xi \in D$, and if so, the value of the jump there equals

$$
[\varphi](\xi)= \begin{cases}\beta_{m, n} & \text { if } \xi=m \kappa+n \sigma \text { and } \tan \theta \text { is irrational, } \\ \sum_{m M+n N=k} \beta_{m, n} & \text { if } \xi=k \nu \text { and } \tan \theta \text { is rational or infinite, }\end{cases}
$$

where the quantities $\beta_{m, n}$ are the Fourier coefficients (4.41). Here we denote

$$
[\varphi](\xi)=\varphi_{+}(\xi)-\varphi_{-}(\xi)
$$

for any $\xi \in \mathbb{R}$, and if $\tan \theta$ is rational or infinite, we have $\nu>0, M$, and $N$ as in (4.44), with $M$ and $N$ having no common factor. In particular, we have at $\xi=0$ that

$$
\varphi_{-}(0)=\frac{1}{2}-\gamma<\frac{1}{2}+\gamma=\varphi_{+}(0),
$$


where $\gamma>0$ is the quantity (4.29).

Proof. Certainly the left- and right-hand limits of $\varphi_{\tau}$ exist and equal $\varphi_{ \pm}(\xi)$, since $\varphi_{\tau}$ is nondecreasing and equals the function $\varphi_{*}$ almost everywhere. With $[\varphi](\xi)$ as in (5.19), and $[h](\xi)=h_{+}(\xi)-h_{-}(\xi)$, we have from (5.9) and the definition (2.4) of $L$ that

$$
(1+4 \alpha)[\varphi](\xi)=\alpha([\varphi](\xi+\kappa)+[\varphi](\xi-\kappa)+[\varphi](\xi+\sigma)+[\varphi](\xi-\sigma))+[h](\xi),
$$

for each $\xi \in \mathbb{R}$.

First suppose that $\xi_{0} \in \mathbb{R} \backslash D$. Then sum both sides of (5.21) over all points $\xi \in D+\xi_{0}$. Each term in the sum is nonnegative, and the sum is finite. Moreover, as $[h](\xi)=0$ for each term in the sum, we obtain $(1+4 \alpha) \Phi=4 \alpha \Phi$ and hence $\Phi=0$, where

$$
\Phi=\sum_{\xi \in D+\xi_{0}}[\varphi](\xi) .
$$

Thus each term in (5.22) is zero, so each $\xi \in D+\xi_{0}$, including $\xi_{0}$, is a point of continuity of $\varphi_{\tau}$. Thus, all discontinuities of $\varphi_{\tau}$ must lie in $D$.

Now suppose that $\tan \theta$ is irrational. Each $\xi \in D$ has a unique representation $\xi=m \kappa+n \sigma$ for integers $m$ and $n$. Denote

$$
\varphi_{m, n}=[\varphi](m \kappa+n \sigma),
$$

multiply both sides of (5.21) at this $\xi$ by $e^{i\left(m \theta_{1}+n \theta_{2}\right)}$ for $\theta_{1}, \theta_{2} \in \mathbb{R}$, and sum over all $m$ and $n$. Denoting

$$
\Phi\left(\theta_{1}, \theta_{2}\right)=\sum_{m=-\infty}^{\infty} \sum_{n=-\infty}^{\infty} \varphi_{m, n} e^{i\left(m \theta_{1}+n \theta_{2}\right)}
$$

which is an absolutely convergent series and hence a continuous function of $\theta_{1}$ and $\theta_{2}$, we obtain

$$
(1+4 \alpha) \Phi\left(\theta_{1}, \theta_{2}\right)=\alpha\left(e^{-i \theta_{1}}+e^{i \theta_{1}}+e^{-i \theta_{2}}+e^{i \theta_{2}}\right) \Phi\left(\theta_{1}, \theta_{2}\right)+1,
$$

where we note that $[h](\xi)=0$, except for $[h](0)=1$. From (5.23) it follows that $\Phi\left(\theta_{1}, \theta_{2}\right)=B\left(\theta_{1}, \theta_{2}\right)$, as in (4.41), and hence that $\varphi_{m, n}=\beta_{m, n}$ for all $m, n$, as claimed in (5.18).

Suppose now that $\tan \theta$ is rational or infinite, and let $\nu, M$, and $N$ be as in the statement of the proposition. Let $\varphi_{k}=[\varphi](k \nu)$ denote the jump at each point $k \nu \in D$, multiply both sides of (5.21) by $e^{i k \theta_{1}}$ for $\theta_{1} \in \mathbb{R}$, and sum over $k$. Letting

$$
\Phi\left(\theta_{1}\right)=\sum_{k=-\infty}^{\infty} \varphi_{k} e^{i k \theta_{1}}
$$

we obtain

$$
(1+4 \alpha) \Phi\left(\theta_{1}\right)=\alpha\left(e^{-i M \theta_{1}}+e^{i M \theta_{1}}+e^{-i N \theta_{1}}+e^{i N \theta_{1}}\right) \Phi\left(\theta_{1}\right)+1,
$$

and this gives

$$
\Phi\left(\theta_{1}\right)=B\left(M \theta_{1}, N \theta_{1}\right)=\sum_{m=-\infty}^{\infty} \sum_{n=-\infty}^{\infty} \beta_{m, n} e^{i(m M+n N) \theta_{1}}
$$


from (4.41). Again, (5.18) easily follows from (5.24).

Finally, (5.20) is a consequence of the formula (5.18) at $\xi=0$, the fact that $\varphi_{-}(0)=1-\varphi_{+}(0)$, which follows from the symmetry relation (5.17) and the formulas (4.43), (4.45), for $\gamma$.

Having constructed the functions

$$
\varphi(\xi)=\varphi_{\tau}\left(\xi, \xi_{0}\right)=\varphi_{\tau}\left(\xi-\xi_{0}\right)
$$

which satisfy (5.14), we now determine the conditions under which these functions satisfy the nonlinear equation

$$
0=\alpha L \varphi(\xi)-f(\varphi(\xi))
$$

for $\xi \in D$, that is, (1.16) with $c=0$. In particular, we determine the relation between the parameters $\tau \in[0,1]$ and $\xi_{0} \in \mathbb{R}$ in (5.25), and the detuning parameter $a$ in the nonlinearity $f$.

Consider $\varphi$ as in (5.25) for some $\tau \in[0,1]$ and $\xi_{0} \in \mathbb{R}$. In order for $\varphi$ to satisfy equation (5.26) for $\xi \in D$, it is necessary and sufficient that

$$
h_{\tau}\left(\xi-\xi_{0}\right) \in h(\varphi(\xi)-a)
$$

for all $\xi \in D$, in view of (5.14) and the formula (3.1) for $f$. We recall here that $h$ is considered as a set-valued function. Now suppose that the inequalities (5.1) hold. Then (5.27) holds for all $\xi \in D \backslash\left\{\xi_{0}\right\}$. Thus if $\xi_{0} \notin D$, the inclusion (5.27) holds for all $\xi \in D$, and the function $\varphi$ satisfies (5.26) for $\xi \in D$. On the other hand, if $\xi_{0} \in D$, then $\varphi$ satisfies (5.26) for $\xi \in D$ if and only if (5.27) holds at $\xi=\xi_{0}$, that is, if and only if

$$
\tau \in h\left(\varphi_{\tau}(0)-a\right) .
$$

At this point it is natural to consider the two cases of rational and irrational slope $\tan \theta$ separately. In the following discussions it will be convenient to identify two solutions of (5.26) which differ by a translation of $\xi$ by an element of $D$. Specifically, if $\varphi(\xi)$ and $\widetilde{\varphi}(\xi)$ both satisfy (5.26) for $\xi \in D$, we shall say these two solutions are equivalent if there exists $\xi_{1} \in D$ such that $\widetilde{\varphi}(\xi)=\varphi\left(\xi+\xi_{1}\right)$ for all $\xi \in D$. By a family of solutions, we shall mean an equivalence class of such solutions, and we shall say that such $\varphi$ and $\widetilde{\varphi}$ belong to the same family.

Case I: $\tan \theta$ is rational or infinite. Let us first observe that, without loss, we may take $\xi_{0} \in D$. Indeed, $\varphi_{\tau}$ is constant on each interval $(n \nu,(n+1) \nu)$ between the points of $D$, by Corollary 5.3. If $\xi_{0} \notin D$, then there exists $\xi_{1} \in D$ such that $\varphi_{\tau}\left(\xi-\xi_{0}\right)=\varphi_{0}\left(\xi-\xi_{1}\right)$ for all $\xi \in D$ (in fact, $\xi_{1}$ is just the point of $D$ nearest to $\xi_{0}$ on the left), so we may replace $\varphi_{\tau}\left(\xi, \xi_{0}\right)$ with $\varphi_{\tau}\left(\xi, \xi_{1}\right)$.

We next see that one way for $(5.28)$ to hold is for

$$
\varphi_{-}(0) \leq a \leq \varphi_{+}(0),
$$

with $\tau \in[0,1]$ uniquely determined so that $\varphi_{\tau}(0)=a$. In this case, the function (5.25) does indeed satisfy the inequalities (5.1), by Theorem 5.1, and so is a solution of (5.26).

Another possibility for $(5.28)$ to hold is that $\varphi_{\tau}(0)<a$, in which case we must have $\tau=0$, and so $\varphi(\xi)=\varphi_{0}\left(\xi, \xi_{0}\right)=\varphi_{-}\left(\xi-\xi_{0}\right)$. The first inequality in (5.1) must 
now hold. The second inequality holds if and only if it holds at $\xi=\xi_{0}+\nu$, that is, if and only if

$$
a<\varphi\left(\xi_{0}+\nu\right)=\varphi_{-}(\nu)=\varphi_{+}(0),
$$

where the final equality in (5.30) is a consequence of the fact that $\varphi_{-}(\xi)=\varphi_{+}(\xi)$ is constant on each interval $(n \nu,(n+1) \nu)$. Thus, we have the solution $\varphi(\xi)=\varphi_{-}\left(\xi-\xi_{0}\right)$ to $(5.26)$, provided that

$$
\varphi_{-}(0)<a<\varphi_{+}(0) .
$$

In a similar fashion, if the strict inequalities (5.31) hold, we also have $\varphi(\xi)=\varphi_{+}\left(\xi-\xi_{0}\right)$ as a solution. However, again in view of the fact that $\varphi$ is constant on each interval $(n \nu,(n+1) \nu)$, the solutions $\varphi_{-}\left(\xi-\xi_{0}\right)$ and $\varphi_{+}\left(\xi-\xi_{0}\right)$ are seen to be equivalent; specifically, we have that $\varphi_{-}\left(\xi-\xi_{0}\right)=\varphi_{+}\left(\xi-\xi_{0}-\nu\right)$ for $\xi \in D$.

Thus if the strict inequality (5.31) holds, we have two distinct families of solutions of (5.26), namely $\varphi(\xi)=\varphi_{\tau}\left(\xi-\xi_{0}\right)$ with $\varphi_{\tau}(0)=a$, and $\varphi(\xi)=\varphi_{ \pm}\left(\xi-\xi_{0}\right)$. At the endpoints of the interval (5.31), that is, for $a=\varphi_{ \pm}(0)$, these two families become the same.

Case II: $\tan \theta$ is irrational. Here we shall obtain uncountably many distinct families of solutions, generally corresponding to the various equivalence classes of $\xi_{0}$ modulo $D$. First suppose that $\xi_{0} \in D$. Then one sees, by arguing as in the case of rational $\tan \theta$, that we obtain three families of solutions to (5.26), namely $\varphi(\xi)=\varphi_{\tau}\left(\xi-\xi_{0}\right)$ with $\varphi_{\tau}(0)=a$, and both $\varphi(\xi)=\varphi_{-}\left(\xi-\xi_{0}\right)$ and $\varphi(\xi)=\varphi_{+}\left(\xi-\xi_{0}\right)$, where in all cases $a$ satisfies (5.29). In contrast to the previous case, however, the families containing $\varphi_{-}\left(\xi-\xi_{0}\right)$ and $\varphi_{+}\left(\xi-\xi_{0}\right)$ are distinct. In particular, the ranges of the functions $\xi \rightarrow \varphi_{-}\left(\xi-\xi_{0}\right)$ and $\xi \rightarrow \varphi_{+}\left(\xi-\xi_{0}\right)$ are disjoint, as a result of the strict monotonicity of $\varphi$, in Theorem 5.1, and so these solutions are not obtained from one another by a translation of $\xi$. Similarly, the solution $\varphi_{\tau}\left(\xi, \xi_{0}\right)$, with $\varphi_{\tau}(0)=a$, is not equivalent to either $\varphi_{ \pm}\left(\xi-\xi_{0}\right)$, except for the obvious relations $\varphi_{0}\left(\xi, \xi_{0}\right)=\varphi_{-}\left(\xi-\xi_{0}\right)$ at $\tau=0$ and $\varphi_{1}\left(\xi, \xi_{0}\right)=\varphi_{+}\left(\xi-\xi_{0}\right)$ at $\tau=1$ when $a=\varphi_{-}(0)$ and $a=\varphi_{+}(0)$, respectively.

When $\xi_{0} \in D$, therefore, we have three distinct families of solutions to equation (5.26) when $a$ satisfies (5.31), and two families at both $a=\varphi_{-}(0)$ and $a=\varphi_{+}(0)$.

Now consider $\xi_{0} \notin D$. In this case, as noted earlier, all we need to do is to verify the inequalities (5.1); the inclusion (5.28) need not be verified. In view of the strict monotonicity of $\varphi$ and the density of $D$, we see that (5.1) holds if and only if $a$ satisfies (5.29). Moreover, Proposition 5.4 implies that the solution $\varphi=\varphi_{\tau}\left(\xi, \xi_{0}\right)$ is independent of $\tau$, since $\xi-\xi_{0} \notin D$ and hence $\varphi_{-}\left(\xi-\xi_{0}\right)=\varphi_{+}\left(\xi-\xi_{0}\right)$ for $\xi \in D$. We finally observe that if $\xi_{0}-\xi_{1} \notin D$, then the solutions $\varphi_{\tau}\left(\xi-\xi_{0}\right)$ and $\varphi_{\tau}\left(\xi-\xi_{1}\right)$ have disjoint ranges for $\xi \in D$ and therefore are not equivalent. Thus, we have distinct families of solutions $\varphi_{\tau}\left(\xi, \xi_{0}\right)$ for distinct values of the parameter $\xi_{0}$, modulo $D$, and this gives uncountably many families of solutions to equation (5.26), for each $a$.

Remark. By (5.20) of Proposition 5.4, we see that we have constructed, for each $a$ satisfying $|a-1 / 2| \leq \gamma$, a class of traveling wave solutions with wave speed $c=0$. This exactly complements the range $\gamma<|a-1 / 2|<1$ for which we have constructed such solutions with wave speed $c \neq 0$. The relation $c=c(a)$ of Theorem 4.7, between the detuning parameter of $f$ in (3.1), and the wave speed $c$, thus extends as a continuous function throughout the range $0<a<1$.

Interestingly, the solutions (5.25) obtained above for the discontinuous nonlinearity $f$ in (3.1) are also solutions for certain continuous nonlinearities $\widetilde{f}$. We take this as partial confirmation of the fact that the traveling wave solutions with speed $c=0$, 
and the phenomenon of propagation failure over a range of parameters is not merely an artifact of the discontinuity in $f$ but rather is a robust phenomenon which one should observe for a large class of nonlinearities.

Specifically, suppose that $\widetilde{f}: \mathbb{R} \rightarrow \mathbb{R}$ is any continuous function satisfying both

$$
\widetilde{f}(u)=u \text { for } u \leq \varphi_{-}(0), \quad \widetilde{f}(u)=u-1 \text { for } u \geq \varphi_{+}(0) .
$$

Then, with $a$ satisfying (5.29) and with $\varphi$ as in (5.25) any of the solutions to (5.26) considered above, we have that

$$
\varphi(\xi) \notin\left(\varphi_{-}(0), \varphi_{+}(0)\right)
$$

and hence that $f(\varphi(\xi))=\tilde{f}(\varphi(\xi))$ for all $\xi \neq \xi_{0}$. In particular, if $\varphi(\xi)=\varphi_{\tau}\left(\xi, \xi_{0}\right)$ is one of the solutions to (5.26) with $\xi_{0} \notin D$, in the case of irrational slope $\tan \theta$, it follows that (5.33) holds for all $\xi \in D$, so $\varphi$ satisfies the equation

$$
0=\alpha L \varphi(\xi)-\widetilde{f}(\varphi(\xi)),
$$

that is, (5.26) with $\tilde{f}$ replacing $f$, for $\xi \in D$. The same holds true of the solutions $\varphi(\xi)=\varphi_{ \pm}\left(\xi-\xi_{0}\right)$ with $\xi_{0} \in D$, since they satisfy (5.33) for all $\xi \in D$, including $\xi=\xi_{0}$.

If $\varphi(\xi)=\varphi_{\tau}\left(\xi, \xi_{0}\right)$ with $\xi_{0} \in D$ and $\tan \theta$ rational, then one sees that $\varphi$ satisfies (5.34) for $\xi \in D$ if and only if (5.34) is satisfied at $\xi=0$, namely, if and only if

$$
\widetilde{f}\left(\varphi_{\tau}(0)\right)=\varphi_{\tau}(0)-\tau .
$$

We use the fact that $\varphi_{\tau}$ satisfies (5.10) in making this conclusion. By (5.32), (5.35) always holds at $\tau=0$ and $\tau=1$, corresponding to $\varphi(\xi)=\varphi_{ \pm}\left(\xi-\xi_{0}\right)$. In addition, if $\widetilde{f}$ is $C^{1}$, then necessarily $\widetilde{f}^{\prime}(0)=\widetilde{f}^{\prime}(1)=1$, which implies that the quantity $\widetilde{f}\left(\varphi_{\tau}(0)\right)-$ $\varphi_{\tau}(0)+\tau$ is positive for $\tau>0$ near 0 , and is negative for $\tau<1$ near 1 . In this case there necessarily exists at least one value $\tau \in(0,1)$ at which $(5.35)$ holds, and this gives a corresponding solution (5.25) to equation (5.34).

Thus, each solution constructed above to the equation (5.26) with the discontinuous nonlinearity $f$ possesses at least one counterpart solution to the equation (5.34) with the smooth nonlinearity $\widetilde{f}$.

6. Conclusions. In this paper we have studied traveling wave solutions for an infinite system of bistable ODEs on a two-dimensional spatial lattice. The traveling waves correspond to a moving planar interface. We have found that there is a range of values of the detuning parameter $a$, which corresponds to a driving force, for which the velocity is zero. This is called propagation failure. The onset of motion of the interface is governed by the normal direction $e^{i \theta}$ of the interface. For each direction, we determine the values $a^{*}=1 / 2 \pm \gamma(\theta)$ of $a$ for which motion of the interface begins. The lowest value of $\gamma(\theta)$ occurs when $\tan \theta$ is irrational; in fact, $\gamma(\theta)$ is shown to be constant and continuous at irrational values of $\tan \theta$ (see (4.43)), and is shown to be discontinuous at rational and infinite values of $\tan \theta$ (see (4.45)).

In Figure 1 the wave speed $c$ is plotted against the detuning parameter $a$, for $\alpha=1$ and for three values of $\theta$, namely for $\tan \theta=0,1$, and 0.1 . Many of the phenomena we have studied are suggested in this figure. In particular, propagation failure clearly manifests itself as a vertical jump in the graph between the values $1 / 2 \pm \gamma(\theta)$, when $c=0$. One further sees that the magnitude of the jump is smallest 


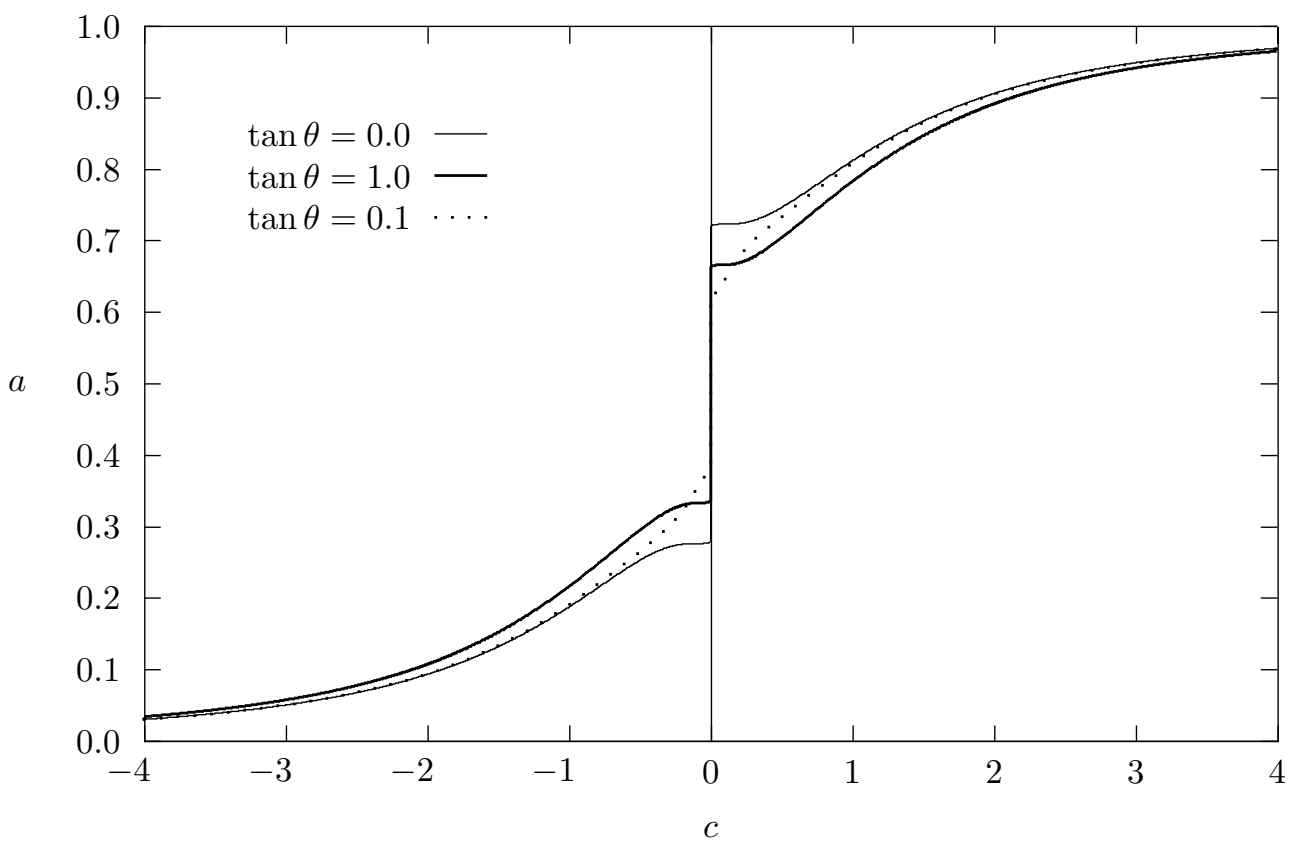

FIG. 1. Graph of wave speed c versus detuning parameter $a$, for $\alpha=1$ and various $\theta$.

when $\tan \theta=1 / 10$ and largest when $\tan \theta=0$. Generally, if $\tan \theta$ is rational, of the form $\tan \theta=p / q$ written in lowest terms, we define the interplanar spacing to be the quantity $\lambda=\left(p^{2}+q^{2}\right)^{-1 / 2}$. If $\tan \theta$ is irrational, then we define $\lambda=0$. The interplanar spacing is the distance between adjacent lines of slope $\tan \theta$ passing through the lattice points. The fraction $1 / 10$ is "nearer" to an irrational (as measured by the smallness of the interplanar spacing) than are the fractions $0 / 1$ or $1 / 1$, so we expect $\gamma(\theta)$ to be smaller there. One also sees the monotone relation between $c$ and $a$ and the limits $a \rightarrow 0,1$ as $c \rightarrow-\infty, \infty$, respectively, are suggested. Indeed, one sees how $c$ is given as a continuous function of $a \in(0,1)$. One can also see that the function $c=c(a, \theta)$ varies continuously in $\theta$ as well (the pathological behavior of $\gamma(\theta)$ noted in section 4 notwithstanding). The proof of this relies on the fact that for fixed $c \neq 0$, the quantity $\Gamma(c)$ in equation (4.26) varies continuously with $\theta$, an immediate consequence of the Lebesgue dominated convergence theorem.

It is possible to conclude from our theory that many of the curves $c=c(a, \theta)$, for various values of $\theta$, must cross in the region where $c \neq 0$. Indeed, one sees such crossings in Figure 1. Quite generally, let $\mathcal{C}(\theta) \subseteq \mathbb{R}^{2}$ denote the curve $c=c(a, \theta)$ in the region $c \neq 0$, namely

$$
\begin{aligned}
\mathcal{C}(\theta) & =\left\{(c, a) \in \mathbb{R}^{2} \mid c=c(a, \theta) \text { with } \gamma(\theta)<|a-1 / 2|<1 / 2\right\} \\
& =\left\{(c, a) \in \mathbb{R}^{2} \mid c \neq 0 \text { and } a=1 / 2+\Gamma(c), \text { with } \Gamma(c) \text { as in }(4.26)\right\} .
\end{aligned}
$$

Now fix two different values $\theta_{0}$ and $\theta_{1}$, for which $\tan \theta_{0}$ and $\tan \theta_{1}$ are distinct rational numbers in the range $[0,1]$. Also fix a positive value of $c=c^{\prime}$ at which the two values $a_{0}$ and $a_{1}$ for which $\left(c^{\prime}, a_{i}\right) \in \mathcal{C}\left(\theta_{i}\right)$ are different. Without loss assume $a_{0}>a_{1}$, so that the curve $\mathcal{C}\left(\theta_{0}\right)$ lies above the curve $\mathcal{C}\left(\theta_{1}\right)$ at $c=c^{\prime}$. Now using the continuous 
dependence of $\Gamma\left(c^{\prime}\right)$ on $\theta$ noted above, we may take a value $\theta=\theta_{2}$ near enough to $\theta_{0}$ so that the value $a=a_{2}$ for which $\left(c^{\prime}, a_{2}\right) \in \mathcal{C}\left(\theta_{2}\right)$ satisfies $a_{2}>a_{1}$. That is, the curve $\mathcal{C}\left(\theta_{2}\right)$ also lies above $\mathcal{C}\left(\theta_{1}\right)$ at $c=c^{\prime}$. On the other hand, we have $\gamma\left(\theta_{2}\right)<\gamma\left(\theta_{1}\right)$ for the limiting values of $\Gamma\left(\theta_{i}\right)$ at $c=0$, for all $\theta_{2}$ near $\theta_{1}$, and so for such $\theta_{2}$ the curve $\mathcal{C}\left(\theta_{2}\right)$ lies below $\mathcal{C}\left(\theta_{1}\right)$ for all small positive values of $c$. Necessarily then, $\mathcal{C}\left(\theta_{2}\right)$ must cross $\mathcal{C}\left(\theta_{1}\right)$ at some positive value of $c$. Indeed, we see the phenomenon described above quite clearly in Figure 1, for the values $\tan \theta_{0}=0, \tan \theta_{1}=1$, and $\tan \theta_{2}=0.1$.

There remains much work to be done on this problem. Generally, the presence of a lattice makes for a much richer and more complex set of dynamical phenomena than would occur in a PDE, such as $u_{t}=\alpha \Delta u-f(u)$, in $\mathbb{R}^{n}$. Although the traveling wave equation (1.7) for this PDE is independent of the dimension $n$, the traveling wave equation for the lattice system (equation (1.16) for the lattice $\mathbb{Z}^{2}$ ) depends not only on the dimension $n$, but also on the underlying lattice. Even though we have restricted our attention in the present paper to the lattices $\mathbb{Z}$ and $\mathbb{Z}^{2}$, it appears that our techniques may be applied to find plane waves in other lattices, for example, in $\mathbb{Z}^{3}$, and in other three-dimensional crystallographic lattices. Such a problem certainly warrants further study.

The stability of the traveling waves needs to be studied. Also, the possibility of multiple traveling waves, and bifurcation of traveling waves, especially for multiple well potentials $F$, is important. A setting in which such problems could be explored is perhaps provided by some of the general theory of mixed differential equations developed in [22]. In particular, a Fredholm alternative for mixed equations is proved there, which would allow the use of the Lyapunov-Schmidt method, and which would place many such problems within the framework of classical bifurcation theory.

Let us also mention the occurrence of spiral waves [31], which have been observed numerically; it would be of interest to understand mathematically how such waves appear. (See also [30] for an analytic treatment of spiral waves, albeit for a different class of problems.)

Finally, for all of the issues dealt with here, it would be interesting to explore the possibility of results in the presence of statistical noise.

In the future, we plan to explore the use of homotopy techniques, both analytically and numerically, to determine the behavior for smooth nonlinearities $f$. For example, the question of both local and global continuation of traveling wave solutions, as parameters in the nonlinearity $f$ are varied, is of significant interest. We mention results of [7] for a class of retarded (time-delay) differential equations, in this spirit, and the recent work in [23] for general mixed differential equations such as (1.16). Such an approach may provide insight into propagation failure for general $f$, and the dependence of this phenomenon on the direction parameter $\theta$.

A significant technical difficulty here is the almost total lack (save for [34], [35], and [22], [23]) of any developed theory for mixed-type functional differential equations, that is, for equations such as (1.16) involving both forward and backward shifts in the argument. One expects some of the many techniques and results of retarded differential equations [18] to carry over, but such a development must proceed with great care.

Let us note here that the study of propagation failure leads to the singular perturbation case $c \rightarrow 0$ of equation (1.16); again, one anticipates that the relatively well-developed theory for retarded differential equations [25] should provide guidelines on how to proceed.

Even for the piecewise linear function $f$ of the present paper, some interesting 
questions remain. For example, what is the asymptotic order of the difference $\delta$ between the quantities $\gamma(\theta)$ for rational (4.45) and irrational (4.43) values of $\tan \theta$ ? We have that

$$
\delta=\frac{1}{2} \sum_{\substack{k=-\infty \\ k \neq 0}}^{\infty} \beta_{-N k, M k},
$$

with $\tan \theta=N / M$, as in (4.44), in lowest terms, and we wish to know the asymptotic behavior of (6.1) as $|M|+|N| \rightarrow \infty$. As Fourier coefficients of an analytic function (4.1), one expects some sort of geometric decay in $\beta_{m, n}$, and so in $\delta$, for large $|M|+|N|$.

Another basic question is to examine the limit $\alpha \rightarrow \infty$, which corresponds to an approximation to the PDE $u_{t}=\Delta u-f(u)$. One expects from the order relation $\alpha=h^{-2}$, where $h$ equals the step size of a discrete approximation, that the magnitude of $c$ should increase like $h^{-1}$, that is, like $\alpha^{-1 / 2}$. The rescaled wave speed $c \alpha^{-1 / 2}$ should approach a limit as $\alpha \rightarrow \infty$. This limit should be independent of $\theta$ and should correspond to the wave speed for the PDE.

In addition to traveling waves with spatially uniform asymptotic states (such as $u_{i} \rightarrow 0$ as $i \rightarrow-\infty$ and $u_{i} \rightarrow 1$ as $i \rightarrow \infty$ ), it is reasonable to study problems with spatially varying states at $\pm \infty$. For example, consider the one-dimensional problem (1.8), where $f$ is a function as in (1.3) or (3.1). With $f(0)=0$, one equilibrium state is $u_{i}=0$ for all $i$, while another is given by

$$
u_{i}= \begin{cases}k_{+}, & i \text { even, } \\ k_{-}, & i \text { odd },\end{cases}
$$

for appropriate quantities $k_{ \pm} \in \mathbb{R}$. In particular, one has that $(\Delta u)_{i}=(-1)^{i} 2\left(k_{-}-\right.$ $\left.k_{+}\right)$, for $u_{i}$ as in (6.2), and equating $\alpha(\Delta u)_{i}$ to $f\left(u_{i}\right)$ yields the required conditions

$$
f\left(k_{+}\right)=-f\left(k_{-}\right)=2 \alpha\left(k_{-}-k_{+}\right) .
$$

For the nonlinearity (3.1), this equation is satisfied by

$$
k_{-}=\frac{2 \alpha}{1+4 \alpha}, \quad k_{+}=\frac{1+2 \alpha}{1+4 \alpha},
$$

provided that $k_{-}<a<k_{+}$. Such an equilibrium solution presents itself as a onedimensional checkerboard. Upon setting

$$
v_{i}=u_{2 i}, \quad w_{i}=u_{2 i+1}
$$

we may rewrite (1.8) as the system

$$
\begin{gathered}
\dot{v}_{i}=\alpha\left(w_{i-1}+w_{i}-2 v_{i}\right)-f\left(v_{i}\right), \\
\dot{w}_{i}=\alpha\left(v_{i}+v_{i+1}-2 w_{i}\right)-f\left(w_{i}\right)
\end{gathered}
$$

and seek traveling wave solutions of (6.3) of the form

$$
v_{i}(t)=\varphi(i-c t), \quad w_{i}(t)=\psi(i-c t)
$$

with boundary conditions

$$
(\varphi(-\infty), \psi(-\infty))=(0,0), \quad(\varphi(\infty), \psi(\infty))=\left(k_{+}, k_{-}\right) .
$$


Such solutions join the zero equilibrium at $-\infty$ to the spatially varying checkerboard equilibrium (6.2) at $\infty$. The functional differential system to be analyzed thus takes the form

$$
\begin{aligned}
& -c \varphi^{\prime}(\xi)=\alpha(\psi(\xi-1)+\psi(\xi)-2 \varphi(\xi))-f(\varphi(\xi)), \\
& -c \psi^{\prime}(\xi)=\alpha(\varphi(\xi)+\varphi(\xi+1)-2 \psi(\xi))-f(\psi(\xi)) .
\end{aligned}
$$

Two-dimensional spatially varying equilibria, such as checkerboards, horizontal and vertical stripes, as well as disordered "chaotic" patterns, are obtained in [10]. One could envision studying the above problem in this more general context.

Quite generally, our knowledge of traveling wave solutions should provide the building blocks for understanding the behavior of more general solutions of the lattice system (1.1). One anticipates that under quite general conditions, the system (1.1) should admit solutions comprised of regions of states close to equilibria, separated by moving interfaces. In general, these interfaces would not be planar but would present themselves as curves in the plane, at least observed on a macroscopic spatial scale of much greater magnitude than the spacing of the lattice points. These curves would move at a speed $c=c(\theta)$ determined locally by the direction angle $\theta$ of the normal vector to the curve. Indeed, such a purely geometrical model, namely, motion of curves determined by normal direction, is studied in [5], and in [37]. For such a model, the limiting shapes (kinetic Wulff shapes) of outward growing interfaces from bounded initial shapes are independent of initial conditions, and connections have been made between such asymptotic shapes and the growth and shapes of crystals. Establishing a rigorous connection between this geometrical model and the lattice system (1.1) is a challenging problem indeed. If our planar results can be extended locally to such a curved interface problem, we predict that kinetic Wulff shapes are composed entirely of rational segments (facets) and that facets on inward growing shapes would shrink and tend to disappear. Smooth inward growing shapes would behave as if $c$ were constant (isotropic) at the value we have found for irrational directions.

\section{REFERENCES}

[1] D. G. Aronson, M. Golubitsky, and J. Mallet-Paret, Ponies on a merry-go-round in a large array of Josephson junctions, Nonlinearity, 4 (1991), pp. 903-910.

[2] J. Bechhoefer, H. Löwen, And L. S. Tuckerman, Dynamical mechanism for the formation of metastable phases, Phys. Rev. Lett., 67 (1991), pp. 1266-1269.

[3] J. W. CAHN, Theory of crystal growth and interface motion in crystalline materials, Acta Metallurgica, 8 (1960), pp. 554-562.

[4] J. W. Cahn, S.-N. Chow, And E. S. VAn Vleck, Spatially discrete nonlinear diffusion equations, Rocky Mountain J. Math., 25 (1995), pp. 87-118.

[5] J. W. CAhn, J. E. TAYlor, ANd C. A. HANDWERKer, Evolving crystal forms: Frank's characteristics revisited, in Sir Charles Frank, OBE, FRS: An Eightieth Birthday Tribute, Hilger, New York, 1991, pp. 88-118.

[6] S.-N. Chow, J. K. Hale, and J. Mallet-Paret, An example of bifurcation to homoclinic orbits, J. Differential Equations, 37 (1980), pp. 351-373.

[7] S.-N. Chow, X.-B. Lin, And J. MALLET-PARET, Transition layers for singularly perturbed delay differential equations with monotone nonlinearities, J. Dynam. Differential Equations, 1 (1989), pp. 3-43.

[8] S.-N. Chow and J. Mallet-Paret, Pattern formation and spatial chaos in lattice dynamical systems: I, IEEE Trans. Circuit Systems, 42 (1995), pp. 746-751.

[9] S.-N. Chow, J. Mallet-Paret, and E. S. Van Vleck, Dynamics of lattice differential equations, Internat. J. Bifur. Chaos Appl. Sci. Engrg., 6 (1996), pp. 1605-1621.

[10] S.-N. Chow, J. Mallet-Paret, and E. S. Van Vleck, Pattern formation and spatial chaos in spatially discrete evolution equations, Random Comput. Dynam., 4 (1996), pp. 109-178. 
[11] S.-N. Chow And W. Shen, Stability and bifurcation of traveling wave solutions in coupled map lattices, Dynam. Systems Appl., 4 (1995), pp. 1-26.

[12] L. O. ChuA And L. YAng, Cellular neural networks: Theory, IEEE Trans. Circuits Systems, 35 (1988), pp. 1257-1272.

[13] L. O. Chua And L. Yang, Cellular neural networks: Applications, IEEE Trans. Circuits Systems, 35 (1988), pp. 1273-1290.

[14] C. E. Elmer And E. S. VAn Vleck, Computation of traveling waves for spatially discrete bistable reaction-diffusion equations, Appl. Numer. Math., 20 (1996), pp. 157-169.

[15] P. C. Fife AND J. B. MCLEOD, The approach of solutions of nonlinear diffusion equations to traveling front solutions, Arch. Rational Mech. Anal., 65 (1977), pp. 335-361.

[16] W. GibBs, The Collected Works of J. Willard Gibbs, Vol. I, Yale University Press, New Haven, CT, 1906.

[17] J. K. Hale And X.-B. Lin, Heteroclinic orbits for retarded functional differential equations, J. Differential Equations, 65 (1986), pp. 175-202.

[18] J. K. Hale and S. M. Verduyn Lunel, Introduction to Functional Differential Equations, Springer-Verlag, 1993.

[19] D. HANKERSON AND B. Zinner, Wavefronts for a cooperative tridiagonal system of differential equations, J. Dynam. Differential Equations, 5 (1993), pp. 359-373.

[20] J. P. KeEner, Propagation and its failure in coupled systems of discrete excitable cells, SIAM J. Appl. Math., 22 (1987), pp. 556-572.

[21] J. Mallet-PARet, Spatial patterns, spatial chaos, and traveling waves in lattice differential equations, in Stochastic and Spatial Structures of Dynamical Systems, S. J. van Strien and S. M. Verdyun Lunel, eds., North-Holland, Amsterdam, 1996, pp. 105-129.

[22] J. MALLET-PARET, The Fredholm alternative for functional differential equations of mixed type, J. Dynam. Differential Equations, to appear.

[23] J. Mallet-PARET, The global structure of traveling waves in spatially discrete dynamical systems, J. Dynam. Differential Equations, to appear.

[24] J. Mallet-Paret and S.-N. Chow, Pattern formation and spatial chaos in lattice dynamical systems: II, IEEE Trans. Circuit Systems, 42 (1995), pp. 752-756.

[25] J. Mallet-Paret and R. D. Nussbaum, Global continuation and asymptotic behavior for periodic solutions of a differential-delay equation, Ann. Mat. Pura Appl., 145 (1986), pp. 33128.

[26] J. Mallet-Paret and R. D. Nussbaum, Multiple transition layers in a singularly perturbed differential-delay equation, Proc. Roy. Soc. Edinburgh Sect. A, 123A (1993), pp. 1119-1134.

[27] R. S. MacKay And J.-A. Sepulchre, Multistability in networks of weakly coupled bistable units, Phys. D, 82 (1995), pp. 243-254.

[28] H. McKean, Nagumo's equation, Adv. Math., 4 (1970), pp. 209-223.

[29] V. K. MeL'NiKov, On the stability of the center for time periodic solutions, Trans. Moscow Math. Soc., 12 (1963), pp. 3-56.

[30] J. E. Paullet And G.B. Ermentrout, Stable rotating waves in two-dimensional discrete active media, SIAM J. Appl. Math., 54 (1994), pp. 1720-1724.

[31] A. Pérez-Muñuzuri, V. Pérez-Muñuzuri, V. Pérez-Villar, and L. O. Chua, Spiral waves on a 2-d array of nonlinear circuits, IEEE Trans. Circuits Systems, 40 (1993), pp. 872-877.

[32] V. Pérez-Muñuzuri, V. Pérez-Villar, and L. O. Chua, Propagation failure in linear arrays of Chua's circuits, Internat. J. Bifur. Chaos Appl. Sci. Engrg., 2 (1992), pp. 403-406.

[33] W. Rudin, Functional Analysis, McGraw-Hill, New York, 1973.

[34] A. Rustichini, Functional differential equations of mixed type: The linear autonomous case, J. Dynam. Differential Equations, 1 (1989), pp. 121-143.

[35] A. Rustichini, Hopf bifurcation for functional differential equations of mixed type, J. Dynam. Differential Equations, 1 (1989), pp. 145-177.

[36] G. R. SelL, Topological Dynamics and Ordinary Differential Equations, Van Nostrand Reinhold, New York, 1971.

[37] J. E. Taylor, J. W. Cahn, and C. A. Handwerker, Geometric models of crystal growth, Acta Metall. Mater., 40 (1992), pp. 1443-1474.

[38] L. S. Tuckerman And J. BechHoefer, Dynamical mechanism for the formation of metastable phases: The case of two nonconserved order parameters, Phys. Rev. A, 46 (1992), pp. 31783192.

[39] H. F. Weinberger, Long-time behavior of a class of biological models, SIAM J. Math. Anal., 13 (1982), pp. 353-396.

[40] J. Wu AND H. XIA, Self-sustained oscillations in a ring array of coupled lossless transmission lines, J. Differential Equations, 124 (1996), pp. 247-278. 
[41] J. Wu AND X. Zou, Patterns of sustained oscillations in neural networks with delayed interactions, J. Appl. Math. Comp., 73 (1995), pp. 55-76.

[42] J. Wu AND X. Zou, Asymptotic and periodic boundary value problems of mixed FDEs and wave solutions of lattice functional differential equations, J. Differential Equations, 135 (1997), pp. 315-357.

[43] B. ZINNER, Stability of traveling wavefronts for the discrete Nagumo equation, SIAM J. Math. Anal., 22 (1991), pp. 1016-1020.

[44] B. ZinNeR, Existence of traveling wavefront solutions for the discrete Nagumo equation, J. Differential Equations, 96 (1992), pp. 1-27.

[45] B. Zinner, G. HARRis, AND W. Hudson, Traveling wavefronts for the discrete Fisher's equation, J. Differential Equations, 105 (1993), pp. 46-62. 